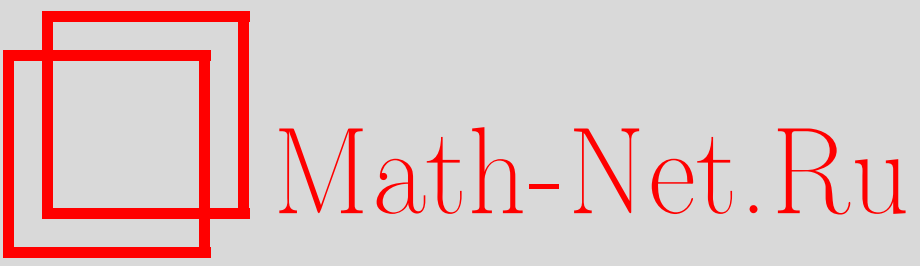

А. В. Атанов, А. В. Лобода, Разложимые пятимерные алгебры Ли в задаче о голоморфной однородности в $\mathbb{C}^{3}$, Итоги науки и техн. Сер. Соврем. мат. и ее прил. Темат. обз., 2019, том 173, 86-115

DOI: https://doi.org/10.36535/0233-6723-2019-173-86-115

Использование Общероссийского математического портала Math-Net.Ru подразумевает, что вы прочитали и согласны с пользовательским соглашением http://www.mathnet.ru/rus/agreement

Параметры загрузки:

IP : 52.205 .19 .152

26 апреля 2023 г., $12: 33: 13$ 


\title{
РАЗЛОЖИМЫЕ ПЯТИМЕРНЫЕ АЛГЕБРЫ ЛИ В ЗАДАЧЕ О ГОЛОМОРФНОЙ ОДНОРОДНОСТИ В $\mathbb{C}^{3}$
}

\author{
(c) 2019 г. $\quad$ A. В. АТАНОВ, А. В. ЛОБОДА
}

\begin{abstract}
АннотАция. В связи с задачей описания голоморфно-однородных вещественных гиперповерхностей пространства $\mathbb{C}^{3}$ изучаются пятимерные вещественные алгебры Ли, реализуемые как алгебры голоморфных векторных полей на таких многообразиях. Доказано, что если на голоморфно однородной вещественной гиперповерхности $M$ пространства $\mathbb{C}^{3}$ имеется разложимая разрешимая пятимерная алгебра Ли голоморфных векторных полей, имеющая полный ранг вблизи некоторой точки $P \in M$, то эта поверхность либо вырождена по Леви (вблизи $P$ ), либо является голоморфным образом аффинно-однородной поверхности.
\end{abstract}

Ключевые слова: однородное многообразие, голоморфное преобразование, разложимая алгебра Ли, векторное поле, вещественная гиперповерхность в $\mathbb{C}^{3}$.

\section{DECOMPOSABLE FIVE-DIMENSIONAL LIE ALGEBRAS IN THE PROBLEM ON HOLOMORPHIC HOMOGENEITY IN $\mathbb{C}^{3}$}

\author{
(c) 2019 A. V. ATANOV, A. V. LOBODA
}

\begin{abstract}
In connection with the problem of describing holomorphically homogeneous real hypersurfaces in the space $\mathbb{C}^{3}$, we study five-dimensional real Lie algebras realized as algebras of holomorphic vector fields on such manifolds. We prove that if on a holomorphically homogeneous real hypersurface $M$ of the space $\mathbb{C}^{3}$, there is a decomposable, solvable, five-dimensional Lie algebra of holomorphic vector fields having a full rank near some point $P \in M$, then this surface is either degenerate near $P$ in the sense of Levy or is a holomorphic image of an affine-homogeneous surface.
\end{abstract}

Keywords and phrases: homogeneous manifold, holomorphic transformation, decomposable Lie algebra, vector field, real hypersurface in $\mathbb{C}^{3}$.

AMS Subject Classification: 32V40, 53B25, 17B66

1. Введение. В настоящей статье изучается задача описания (локально) однородных вещественных гиперповерхностей трехмерного комплексного пространства. Базируется такое изучение на рассмотрении пятимерных алгебр Ли голоморфных векторных полей, касательных к однородным гиперповерхностям. Сами такие поверхности предполагаются невырожденными по Леви (в некоторой, а значит, и в любой близкой точке).

Обозначенные ограничения на размерность алгебр Ли, отвечающих однородным поверхностям, а также требование Леви-невырожденности изучаемых многообразий фактически не являются ограничениями в сегодняшнем состоянии исследуемой задачи. Они лишь отделяют ее нерешенные фрагменты от уже изученных.

Работа А. В. Лободы выполнена при поддержке Российского фонда фундаментальных исследований (проект № 17-01-00592-a). 
Уточним в связи с этим, что однородные вырожденные по Леви гиперповерхности пространства $\mathbb{C}^{3}$ полностью описаны в [22]. Для так называемых сферических гиперповерхностей, являющихся голоморфными образами двух (невырожденных по Леви) квадрик

$$
v=\left|z_{1}\right|^{2}+\left|z_{2}\right|^{2}, \quad v=\left|z_{1}\right|^{2}-\left|z_{2}\right|^{2}
$$

в $\mathbb{C}^{3}$, размерности транзитивно действующих на них групп Ли (а следовательно, и соответствующих алгебр Ли) равны 15 (см. [18]). Размерность же локальной группы $G(M)$ голоморфных преобразований, транзитивно действующей на несферической невырожденной гиперповерхности $M \subset \mathbb{C}^{3}$, удовлетворяет (см. [8]) двусторонней оценке $5 \leqslant \operatorname{dim}_{\mathbb{R}} G(M) \leqslant 8$.

При этом все однородные поверхности с семимерными и восьмимерными алгебрами Ли описаны в $[9,10] ;$ случай шестимерных алгебр полностью изучен в $[21]$.

Подход, используемый в статье, связан с реализацией абстрактных пятимерных алгебр Ли в виде алгебр голоморфных векторных полей в пространстве $\mathbb{C}^{3}$. Необходимым и достаточным условием для того, чтобы такая алгебра имела в качестве интегрального многообразия именно пятимерную вещественную (голоморфно-однородную) гиперповерхность, является, в силу теоремы Фробениуса (см. [4]), полнота ранга этой алгебры.

В [16] предложена техническая идея перехода от произвольной абстрактной пятимерной алгебры Ли к соответствующим этой алгебре однородным поверхностям. Именно эта идея является стержнем настоящей статьи. Отметим, однако, что количество алгебраически различных пятимерных алгебр достаточно велико: в списке $[13,14]$ (см. также [7]) содержится 67 типов таких алгебр, при этом многие типы включают в себя не только отдельные (точечные) алгебры, но и семейства алгебр, зависящие от параметров (в количестве от одного до трех).

Поэтому оправданным в заявленной задаче является изучение не всего множества пятимерных алгебр Ли, а их отдельных совокупностей. Так, в [1] изучены голоморфные реализации пятимерных нильпотентных алгебр Ли и описаны однородные (Леви-невырожденные) гиперповерхности в $\mathbb{C}^{3}$, отвечающие таким алгебрам. Нильпотентных алгебр имеется девять, а соответствующих им невырожденных поверхностей оказалось (с точностью до голоморфной эквивалентности) всего две: положительно определенная и индефинитная квадрики (1.1).

Целью настоящей работы является изучение еще одного естественного семейства пятимерных алгебр Ли, а именно, разложимых алгебр. В списке [14] таких алгебр имеется 27, а потому потребовалась выработка дополнительных обобщающих подходов к изучению не отдельных алгебр, а подсемейств обозначенного достаточно обширного семейства. Авторы выражают благодарность И. Г. Коссовскому за обсуждение таких идей и подходов, с помощью которых была практически полностью реализована поставленная задача о 27 алгебрах.

В то же время в настоящую статью не вошло описание неразрешимых разложимых пятимерных алгебр Ли. Изучение авторами голоморфных реализаций всех таких четырех алгебр практически завершено, однако развернутое описание соответствующих результатов (анонсированных в [2]) планируется изложить в отдельной работе.

Сформулируем в обобщенной форме основной результат настоящей статьи.

Теорема 1.1. Если на голоморфно однородной вещественной гиперповерхности М пространства $\mathbb{C}^{3}$ имеется разложимая разрешимая пятимерная алгебра Ли голоморфных векторных полей, имеющая полный ранг вблизи некоторой точки $P \in M$, то эта поверхность либо вырождена по Леви (вблизи Р), либо является голоморфным образом афбинно-однородной поверхности.

Отметим, что свойство аффинной однородности в этой формулировке можно заменить трубчатостью. Напомним в связи с этим, что в двумерном случае (см. [11]) и в большинстве известных примеров в $\mathbb{C}^{3}$ голоморфная однородность трубчатой поверхности означает аффинную (вещественную) однородность ее основания. При этом среди голоморфно-однородных поверхностей с рассмотренными в статье алгебрами Ли имеется семейство, для всех представителей которого трубчатость и аффинная однородность достигаются в разных координатных системах. 
Еще одним свойством изученных разложимых пятимерных алгебр является наличие в них «скрытых симметрий»: каждая невырожденная орбита такой алгебры имеет размерность полной алгебры симметрий, строго большую чем 5.

Приведенная формулировка теоремы 1.1 и комментарии к ней являются следствиями описания (см. теоремы 5.1 и 6.1, а также предложения 4.1 и 7.1) всех однородных невырожденных поверхностей, ассоциированных с каждой из обсуждаемых алгебр. Еще одной переформулировкой основного результата, вытекающей из проведенного исследования, является утверждение об отсутствии новых однородных поверхностей, связанных с изученным семейством алгебр. Это утверждение и результат работы [1] в некотором смысле противоположны выводу о «большом количестве» однородных гиперповерхностей в $\mathbb{C}^{3}$, фактически обозначенному в [16].

В целом семейство таких поверхностей достаточно велико, но в рамках развиваемого в настоящей статье подхода вполне реальным является получение полного описания этого семейства в ближайшей перспективе.

2. Список пятимерных разложимых алгебр Ли. Классификационная статья Г. М. Мубаракзянова [14] о пятимерных алгебрах Ли начинается со списка 27 типов разложимых алгебр. Каждая из них содержит несколько маломерных слагаемых и имеет соответствующий условный номер, ссылающийся на другую статью [13] того же автора. Ниже мы приводим в табличной форме явное описание всех этих алгебр.

Каждая строка таблицы 1 представляет собой набор коммутационных соотношений для соответствующего типа алгебр. Элементы $e_{1}, e_{2}, e_{3}, e_{4}, e_{5}$ представляют собой базис каждой абстрактной алгебры Ли, коммутатор двух (базисных) элементов $e_{i}, e_{j}$ алгебры обозначается традиционной квадратной скобкой $\left[e_{i}, e_{j}\right]$. Пустая клетка для коммутатора $\left[e_{i}, e_{j}\right]$ означает, что он равен нулю.

Параметры, присутствующие в коммутационных соотношениях некоторых алгебр, удовлетворяют следующим ограничениям:

$$
\begin{array}{ll}
\mathfrak{m}_{7}, \mathfrak{m}_{14}: & -1 \leqslant s<1, s \neq 0, \\
\mathfrak{m}_{8}, \mathfrak{m}_{15}, \mathfrak{m}_{23}, \mathfrak{m}_{26}: & p \geqslant 0, \\
\mathfrak{m}_{19}, \mathfrak{m}_{23}: & \alpha \neq 0, \\
\mathfrak{m}_{22}: & -1 \leqslant \gamma \leqslant \beta \leqslant 1, \beta \gamma \neq 0, \\
\mathfrak{m}_{25}: & |q| \leqslant 1 .
\end{array}
$$

Для упорядочения отдельных типов алгебр мы снабжаем их номерами в сплошной нумерации от $\mathfrak{m}_{1}$ до $\mathfrak{m}_{27}$. Соответствие этих номеров и типов алгебр из списка [14] устанавливается следующим образом:

$$
\begin{aligned}
& \mathfrak{m}_{1}=5 g_{1}, \quad \mathfrak{m}_{2}=g_{2}+3 g_{1}, \quad \mathfrak{m}_{3}=2 g_{2}+g_{1}, \\
& \mathfrak{m}_{j}=g_{3, j-3}+2 g_{1} \quad(j=4, \ldots, 10), \\
& \mathfrak{m}_{k}=g_{3, k-10}+g_{2} \quad(k=11, \ldots, 17), \\
& \mathfrak{m}_{l}=g_{4, l-17}+g_{1} \quad(l=18, \ldots, 27) .
\end{aligned}
$$

После установления такого соответствия все обсуждения статьи строятся только на базе таблицы 1. Отметим сразу, что из внесенных в нее типов четыре алгебры

$$
\mathfrak{m}_{9}, \quad \mathfrak{m}_{10}, \quad \mathfrak{m}_{16}, \quad \mathfrak{m}_{17}
$$

являются неразрешимыми. Эти алгебры, как было сказано, мы исключаем из рассмотрения в настоящей работе.

3. Схема исследования голоморфной однородности. При обсуждении реализаций произвольной пятимерной алгебры Ли с базисом $e_{1}, e_{2}, e_{3}, e_{4}, e_{5}$ в виде алгебры голоморфных векторных полей в $\mathbb{C}^{3}$ будем записывать каждый элемент этого базиса в виде

$$
e_{k}=f_{k}\left(z_{1}, z_{2}, w\right) \frac{\partial}{\partial z_{1}}+g_{k}\left(z_{1}, z_{2}, w\right) \frac{\partial}{\partial z_{2}}+h_{k}\left(z_{1}, z_{2}, w\right) \frac{\partial}{\partial w}, \quad k=1, \ldots, 5,
$$


Таблица 1. Классификация пятимерных разложимых алгебр Ли (см. [13,14])

\begin{tabular}{|c|c|c|c|c|c|c|c|c|c|c|}
\hline 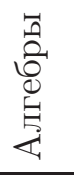 & {$\left[e_{1}, e_{2}\right]$} & {$\left[e_{1}, e_{3}\right]$} & {$\left[e_{1}, e_{4}\right]$} & {$\left[e_{1}, e_{5}\right]$} & {$\left[e_{2}, e_{3}\right]$} & {$\left[e_{2}, e_{4}\right]$} & {$\left[e_{2}, e_{5}\right]$} & {$\left[e_{3}, e_{4}\right]$} & {$\left[e_{3}, e_{5}\right]$} & {$\left[e_{4}, e_{5}\right]$} \\
\hline \multicolumn{11}{|l|}{$\mathfrak{m}_{1}$} \\
\hline $\mathfrak{m}_{2}$ & $e_{1}$ & & & & & & & & & \\
\hline $\mathfrak{m}_{3}$ & $e_{1}$ & & & & & & & $e_{3}$ & & \\
\hline $\mathfrak{m}_{4}$ & & & & & $e_{1}$ & & & & & \\
\hline $\mathfrak{m}_{5}$ & & $e_{1}$ & & & $e_{1}+e_{2}$ & & & & & \\
\hline $\mathfrak{m}_{6}$ & & $e_{1}$ & & & $e_{2}$ & & & & & \\
\hline $\mathfrak{m}_{7}$ & & $e_{1}$ & & & $s e_{2}$ & & & & & \\
\hline $\mathfrak{m}_{8}$ & & $p e_{1}-e_{2}$ & & & $e_{1}+p e_{2}$ & & & & & \\
\hline $\mathfrak{m}_{9}$ & $e_{1}$ & $2 e_{2}$ & & & $e_{3}$ & & & & & \\
\hline $\mathfrak{m}_{10}$ & $e_{3}$ & $-e_{2}$ & & & $e_{1}$ & & & & & \\
\hline $\mathfrak{m}_{11}$ & & & & & $e_{1}$ & & & & & $e_{4}$ \\
\hline $\mathfrak{m}_{12}$ & & $e_{1}$ & & & $e_{1}+e_{2}$ & & & & & $e_{4}$ \\
\hline $\mathfrak{m}_{13}$ & & $e_{1}$ & & & $e_{2}$ & & & & & $e_{4}$ \\
\hline $\mathfrak{m}_{14}$ & & $e_{1}$ & & & $s e_{2}$ & & & & & $e_{4}$ \\
\hline $\mathfrak{m}_{15}$ & & $p e_{1}-e_{2}$ & & & $e_{1}+p e_{2}$ & & & & & $e_{4}$ \\
\hline $\mathfrak{m}_{16}$ & $e_{1}$ & $2 e_{2}$ & & & $e_{3}$ & & & & & $e_{4}$ \\
\hline $\mathfrak{m}_{17}$ & $e_{3}$ & $-e_{2}$ & & & $e_{1}$ & & & & & $e_{4}$ \\
\hline $\mathfrak{m}_{18}$ & & & & & & $e_{1}$ & & $e_{2}$ & & \\
\hline $\mathfrak{m}_{19}$ & & & $\alpha e_{1}$ & & & $e_{2}$ & & $e_{2}+e_{3}$ & & \\
\hline $\mathfrak{m}_{20}$ & & & $e_{1}$ & & & & & $e_{2}$ & & \\
\hline $\mathfrak{m}_{21}$ & & & $e_{1}$ & & & $e_{1}+e_{2}$ & & $e_{2}+e_{3}$ & & \\
\hline $\mathfrak{m}_{22}$ & & & $e_{1}$ & & & $\beta e_{2}$ & & $\gamma e_{3}$ & & \\
\hline $\mathfrak{m}_{23}$ & & & $\alpha e_{1}$ & & & $p e_{2}-e_{3}$ & & $e_{2}+p e_{3}$ & & \\
\hline $\mathfrak{m}_{24}$ & & & $2 e_{1}$ & & $e_{1}$ & $e_{2}$ & & $e_{2}+e_{3}$ & & \\
\hline $\mathfrak{m}_{25}$ & & & $(1+q) e_{1}$ & & $e_{1}$ & $e_{2}$ & & $q e_{3}$ & & \\
\hline $\mathfrak{m}_{26}$ & & & $2 p e_{1}$ & & $e_{1}$ & $p e_{2}-e_{3}$ & & $e_{2}+p e_{3}$ & & \\
\hline $\mathfrak{m}_{27}$ & & $e_{1}$ & $-e_{2}$ & & $e_{2}$ & $e_{1}$ & & & & \\
\hline
\end{tabular}

с голоморфными (вблизи обсуждаемой точки поверхности) функциональными коэффициентами $f_{k}, g_{k}, h_{k}$. Будем использовать также записи вида $e_{k}=\left(f_{k}, g_{k}, h_{k}\right)$ для сокращения формулы (3.1).

Через $z_{1}, z_{2}, w$ здесь и далее обозначаются координаты в пространстве $\mathbb{C}^{3}$. Их вещественные и мнимые части будем обозначать через $x_{k}=\operatorname{Re} z_{k}, y_{k}=\operatorname{Im} z_{k}, k=1,2, u=\operatorname{Re} w, v=\operatorname{Im} w$. Пару координат $\left(z_{1}, z_{2}\right)$ мы часто будем объединять в двумерный комплексный вектор $z$.

Свойство однородности вещественной пятимерной гиперповерхности $M \subset \mathbb{C}^{3}$ всюду в статье обсуждается в локальном смысле и понимается как наличие на такой поверхности вещественной алгебры Ли $g(M)$ голоморфных (касательных к $M$ ) векторных полей, имеющей вблизи обсуждаемой точки ранг, равный 5. При этом обсуждаемую точку однородной поверхности $M$ будем считать совпадающей с началом координат пространства $\mathbb{C}^{3}$. 
Сопутствующая задача, решаемая в процессе голоморфной реализации конкретной алгебры Ли, - получить за счет голоморфных преобразований по возможности «простой» вид функциональных коэффициентов $f_{k}, g_{k}, h_{k}$ для всей базисной пятерки полей.

Напомним, что согласно «основной теореме» теории обыкновенных дифференциальных уравнений (справедливой и в голоморфном случае), любое гладкое векторное поле можно выпрямить вблизи неособой точки подходящим координатным диффеоморфизмом. Всюду ниже мы считаем, что базисное поле $e_{1}$ имеет упрощенный (выпрямленный) вид $e_{1}=\partial / \partial w$. Коэффициенты такого выпрямленного поля являются константами и не зависят ни от каких координат трехмерного пространства, в котором это поле рассматривается.

Используя технику работы [16], развивающей идеи Э. Картана (см. [17]), мы будем упрощать несколько базисных полей одновременно. Одна из возможностей таких упрощений - уменьшение количества аргументов у функциональных коэффициентов $f_{k}, g_{k}, h_{k}$ полей вида (3.1). Во многих обсуждениях статьи удается доводить наборы аргументов у таких функций до двух переменных (например, до $\left.z=\left(z_{1}, z_{2}\right)\right)$ или до одной. В последнем случае в роли такой единственной переменной, как правило, выступает $z_{2}$, а соответствующие функции мы обозначаем через $\hat{f}_{k}\left(z_{2}\right), \hat{g}_{k}\left(z_{2}\right)$, $\hat{h}_{k}\left(z_{2}\right)$.

При этом мы обсуждаем в статье только невырожденные по Леви поверхности. Определяющие функции таких поверхностей зависят от всех трех комплексных переменных и, более того, матрица Гессе вторых производных, суженная на комплексную касательную плоскость к такой поверхности, является невырожденной эрмитовой матрицей.

Для алгебр векторных полей на однородных поверхностях оказываются справедливыми следующие свойства, связанные с одновременным упрощением или выпрямлением нескольких базисных полей.

Лемма 3.1. Если на невырожденной по Леви гиперповерхности $M \subset \mathbb{C}^{3}$ имеется пара коммутирующих голоморфных векторных полей е $e_{1}$ и е $e_{2}$, линейно независимых над $\mathbb{R}$, то голоморфной заменой координат эта пара может быть выпрямлена, т.е. приведена (вблизи некоторой точки поверхности) к виду

$$
e_{1}=(0,0,1), \quad e_{2}=(1,0,0)
$$

Лемма 3.2. Пусть $M \subset \mathbb{C}^{3}$ - вещественно-аналитическал гиперповерхность, невырожденная по Леви, $g(M)$ - пятимерная алгебра касательных $к M$ голоморфных векторных полей, имеющал полный ранг (вблизи некоторой точки поверхности $M), g^{*}(M)=\left\langle e_{i}, e_{j}, e_{k}\right\rangle-$ трехмерная абелева подалгебра в $g(M)$. Тогда голоморфным преобразованием базис этой подалгебры можно привести (вблизи некоторой точки поверхности $M$ ) к одному из двух видов:

$$
\begin{array}{ll}
\text { (1) } \quad e_{i}=(1,0,0), \quad e_{j}=(0,1,0), & e_{k}=(0,0,1), \\
\text { (2) } \quad e_{i}=(1,0,0), & e_{j}=(0,0,1), \quad e_{k}=\left(\hat{f}_{k}\left(z_{2}\right), 0, \hat{h}_{k}\left(z_{2}\right)\right) .
\end{array}
$$

Лемма 3.3 (И. Г. Коссовский). Пусть в пятимерной алгебре $g(M)$ голоморфных векторных полей на вещественной гиперповерхности $M \subset \mathbb{C}^{3}$ имеется четырехмерная абелева подалгебра. Тогда М вырождена по Леви.

Лемма 3.4. Если четверка базисных голоморфных полей пятимерной алгебры $g(M)$ полного ранга имеет вблизи некоторой точки $M$ вид

$$
\begin{aligned}
& e_{1}=\left(f_{1}\left(z_{1}, z_{2}, z_{3}\right), 0, h_{1}\left(z_{1}, z_{2}, z_{3}\right)\right), \\
& e_{2}=\left(f_{2}\left(z_{1}, z_{2}, z_{3}\right), 0, h_{2}\left(z_{1}, z_{2}, z_{3}\right)\right), \\
& e_{3}=\left(f_{3}\left(z_{1}, z_{2}, z_{3}\right), 0, h_{3}\left(z_{1}, z_{2}, z_{3}\right)\right), \\
& e_{4}=\left(f_{4}\left(z_{1}, z_{2}, z_{3}\right), 0, h_{4}\left(z_{1}, z_{2}, z_{3}\right)\right),
\end{aligned}
$$

то поверхность М является вырожденной по Леви (вблизи обсуждаемой точки). 
Ниже мы приводим доказательства этих фактов. Отметим при этом естественную перестановку в порядке следования доказательств, так как утверждение леммы 3.4 является одним из аргументов при обсуждении леммы 3.3. Кроме того, зафиксируем в виде замечаний два технических утверждения из [16], необходимых для доказательства сформулированных лемм.

Замечание 3.1. Если вблизи некоторой точки пространства $\mathbb{C}^{3}$ заданы два голоморфных векторных поля $e_{1}=(0,0,1)$ и $e_{2}=\left(f_{2}\left(z_{1}, z_{2}\right), g_{2}\left(z_{1}, z_{2}\right), h_{2}\left(z_{1}, z_{2}\right)\right)$ и пара $\left(f_{2}, g_{2}\right)$ не обращается в нуль в обсуждаемой точке, то голоморфной заменой координат можно выпрямить поле $e_{2}$ вблизи этой точки (например, до вида $\left.e_{2}=(1,0,0)\right)$ с сохранением выпрямленного поля $e_{1}$.

Замечание 3.2. Назовем голоморфное векторное поле $Z$ в пространстве $\mathbb{C}^{3}$ линеаризованным no паре переменных $z_{1}, w$ (в обсуждаемой системе координат), если оно имеет вид

$$
Z=\left(\alpha z_{1}+\beta w+\hat{f}\left(z_{2}\right), \hat{g}\left(z_{2}\right), \gamma z_{1}+\delta w+\hat{h}\left(z_{2}\right)\right)
$$

с некоторыми голоморфными функциями $\hat{f}, \hat{g}, \hat{h}$ и комплексными константами $\alpha, \beta, \gamma, \delta$.

В [16] доказано утверждение о существовании (локальной) голоморфной замены, сохраняющей пару выпрямленных полей $e_{1}=(0,0,1), e_{2}=(1,0,0)$ и упрощающей линеаризованное поле

до состояния

$$
e_{3}=\left(l\left(z_{1}, w\right)+\hat{f}_{3}\left(z_{2}\right), \hat{g}_{3}\left(z_{2}\right), L\left(z_{1}, w\right)+\hat{h}_{3}\left(z_{2}\right)\right)
$$

$$
e_{3}=\left(l\left(z_{1}, w\right), 1, L\left(z_{1}, w\right)\right)
$$

с теми же линейными функциями $l\left(z_{1}, w\right), L\left(z_{1}, w\right)$.

Достаточным условием существования такой замены является неравенство $\hat{g}_{3} \neq 0$ вблизи обсуждаемой точки. Отметим также, что эта замена сохраняет линейные функции $\alpha z_{1}+\beta w, \gamma z_{1}+\delta w$ и вид (3.3) (с измененными функциями $\left.\hat{f}\left(z_{2}\right), \hat{g}\left(z_{2}\right), \hat{h}\left(z_{2}\right)\right)$ любого другого поля, линеаризованного по паре переменных $z_{1}, w$.

Доказательство леммы 3.1. Первое из двух обсуждаемых в лемме полей можно сразу считать имеющим вид

$$
e_{1}=(0,0,1)=\frac{\partial}{\partial w} \text {. }
$$

В силу коммутирования полей $e_{1}$ и $e_{2}$ компоненты $f_{2}\left(z_{1}, z_{2}, w\right), g_{2}\left(z_{1}, z_{2}, w\right), h_{2}\left(z_{1}, z_{2}, w\right)$ второго поля

$$
e_{2}=f_{2} \frac{\partial}{\partial z_{1}}+g_{2} \frac{\partial}{\partial z_{2}}+h_{2} \frac{\partial}{\partial w}
$$

не зависят от переменной $w$.

Рассмотрим теперь два случая, связанные с парой компонент $f_{2}\left(z_{1}, z_{2}\right), g_{2}\left(z_{1}, z_{2}\right)$ поля $e_{2}$.

Первый случай, когда обе они тождественно нулевые (вблизи некоторой точки $M$ ), означает наличие на поверхности $M$ двух линейно независимых над $\mathbb{R}$ полей вида

$$
e_{1}=\left(0,0, h_{1}\left(z_{1}, z_{2}, w\right)\right), \quad e_{2}=\left(0,0, h_{2}\left(z_{1}, z_{2}, w\right)\right) .
$$

Для таких полей (3.4) определитель

$$
\left|\begin{array}{ll}
\operatorname{Re} h_{1} & \operatorname{Im} h_{1} \\
\operatorname{Re} h_{2} & \operatorname{Im} h_{2}
\end{array}\right|
$$

отличен от нуля. Условия же касания полями $e_{1}, e_{2}$ обсуждаемой поверхности $M$ означают выполнение двух равенств

$$
\operatorname{Re}\left(\left.h_{1} \frac{\partial}{\partial w} \Phi\left(z_{1}, z_{2}, w\right)\right|_{M}\right)=0, \quad \operatorname{Re}\left(\left.h_{2} \frac{\partial}{\partial w} \Phi\left(z_{1}, z_{2}, w\right)\right|_{M}\right)=0 .
$$

Отсюда следует тождественное равенство нулю производных $\partial \Phi / \partial u$ и $\partial \Phi / \partial v$, т.е. независимость определяющей функции $\Phi\left(z_{1}, z_{2}, w\right)$ от переменной $w$ и, следовательно, вырожденность по Леви обсуждаемой поверхности $M$. 
Тем самым, единственно возможным для невырожденной по Леви поверхности является второй случай, в рамках которого на поверхности $M$ найдется точка, где хотя бы одна из пары функций $f_{2}\left(z_{1}, z_{2}\right), g_{2}\left(z_{1}, z_{2}\right)$ отлична от нуля. Завершает доказательство леммы 3.1 ссылка на замечание 3.1 .

Доказательство леммы 3.2. Пользуясь леммой 3.1, выпрямим два базисных поля подалгебры $g^{*}(M)$ до состояния

$$
e_{i}=(1,0,0), \quad e_{j}=(0,0,1) .
$$

Тогда в силу равенства нулю коммутаторов $\left[e_{i}, e_{k}\right]$ и $\left[e_{j}, e_{k}\right]$ компоненты $f_{k}, g_{k}, h_{k}$ третьего базисного поля

$$
e_{k}=\left(f_{k}, g_{k}, h_{k}\right)=f_{k} \frac{\partial}{\partial z_{1}}+g_{k} \frac{\partial}{\partial z_{2}}+h_{k} \frac{\partial}{\partial w}
$$

этой подалгебры могут зависеть только от переменной $z_{2}$. Если при этом компонента $g_{2}\left(z_{2}\right)$ не является тождественно нулевой вблизи некоторой точки поверхности, то (вблизи этой точки) согласно замечанию 3.2 это третье поле также можно выпрямить до состояния $e_{k}=(0,1,0)$. Выпрямленный вид (3.5) двух первых полей также сохраняется. Такая ситуация соответствует случаю 1 леммы 3.2 .

Если же компонента $g_{2}\left(z_{2}\right)$ тождественно нулевая вблизи некоторой точки поверхности, то мы получаем случай 2 доказываемой леммы.

Доказательство леммы 3.4. Обозначим через $\Phi\left(z_{1}, z_{2}, w\right)$ определяющую функцию интегрального многообразия $M=\{\Phi=0\}$ алгебры $g$, проходящего через начало координат. Условия касания вещественной гиперповерхности $M$ базисными полями алгебры $g$ имеют вид

$$
\operatorname{Re}\left(\left.e_{k}(\Phi)\right|_{M}\right) \equiv 0, \quad k=1,2,3,4,5 .
$$

Например, для поля $e_{1}$ получаем

$$
\operatorname{Re}\left(f_{1} \frac{\partial \Phi}{\partial z_{1}}+h_{1} \frac{\partial \Phi}{\partial w}\right)=0
$$

или в развернутой форме

$$
\operatorname{Re}\left(f_{1}\right) \frac{\partial \Phi}{\partial x_{1}}+\operatorname{Im}\left(f_{1}\right) \frac{\partial \Phi}{\partial y_{1}}+\operatorname{Re}\left(h_{1}\right) \frac{\partial \Phi}{\partial u}+\operatorname{Im}\left(h_{1}\right) \frac{\partial \Phi}{\partial v}=0 .
$$

Аналогичным развернутым образом можно записать и остальные четыре условия (3.6). Совокупность первых четырех таких равенств будем рассматривать как систему линейных уравнений относительно производных

$$
\operatorname{Re}\left(\frac{\partial \Phi}{\partial z_{1}}\right), \quad \operatorname{Im}\left(\frac{\partial \Phi}{\partial z_{1}}\right), \quad \operatorname{Re}\left(\frac{\partial \Phi}{\partial w}\right), \quad \operatorname{Im}\left(\frac{\partial \Phi}{\partial w}\right) .
$$

Коэффициентами системы являются наборы координат первых четырех полей (3.2). Условие полноты ранга этого набора полей означает, что такие координаты образуют невырожденную (вблизи выделенной точки) $(4 \times 4)$-матрицу. Тогда интересующие нас производные тождественно равны нулю вблизи обсуждаемой точки поверхности $M$. Это означает, что интегральная поверхность $M$ вырождена по Леви. Лемма 3.4 доказана.

Доказательство леммы 3.3. Предположим, что поверхность $M$ невырожденная и рассмотрим произвольную независимую над $\mathbb{R}$ тройку полей $e_{1}, e_{2}, e_{3}$ из четырехмерной абелевой подалгебры $T$ исходной алгебры.

Согласно лемме 3.2 либо (I) вся эта тройка выпрямляется, либо (II) выпрямляется только пара полей $e_{1}, e_{2}$, а третье при этом примет вид $\left(\hat{f}_{3}, 0, \hat{h}_{3}\right)$.

В первом случае коммутирование четвертого базисного поля $e_{4}$ подалгебры $T$ с тремя выпрямленными полями означает, что все компоненты $e_{4}-$ константы. Рассматривая вместо $e_{4}$ линейную комбинацию этого поля с полями $e_{1}, e_{2}, e_{3}$, можно получить в подалгебре $T$ элемент $e_{4}^{*}=(i a, i b, i c)$ с чисто мнимыми постоянными компонентами.

Но и поле $e_{1}^{*}=(a, b, c)$ также является касательным к $M$. Выпрямляя теперь поле $e_{1}^{*}$ до состояния $\partial / \partial w$, получим в новых координатах $e_{4}^{*}=i \partial / \partial w$. Наличие на поверхности двух таких 
полей означает независимость ее уравнения от переменной $w$, а значит, вырождение по Леви в противоречии с допущением о невырожденности.

В условиях второго случая рассмотрим пару выпрямленных полей $e_{1}=\partial / \partial z_{1}, e_{2}=\partial / \partial w$, поле $e_{3}=\left(f_{3}, g_{3}, h_{3}\right)$ и линейную оболочку $V=\left\langle e_{1}, e_{2}, e_{3}\right\rangle$.

Компоненты любого поля $e_{4}=\left(f_{4}, g_{4}, h_{4}\right)$ из $T \backslash V$ зависят (в силу его коммутирования с полями $\left.e_{1}, e_{2}\right)$ не более чем от переменной $z_{2}$. Если бы в $T \backslash V$ нашлось поле, для которого $g_{4}\left(z_{2}\right) \neq 0$, то согласно замечанию 3.2 такое поле можно выпрямить с сохранением полей $e_{1}, e_{2}$. Выше показано, что первый случай, к которому свелась такая ситуация, противоречит невырожденности.

В рамках же второго случая все поля из $T$ имеют тождественно нулевую вторую компоненту. Но в силу леммы 3.4 наличие такой четверки полей, касательных к поверхности $M$, снова означает ее вырожденность по Леви. Лемма 3.3 доказана.

Теперь обозначим схему исследования (с использованием доказанных лемм) однородных невырожденных по Леви поверхностей, отвечающих разложимым неразрешимым алгебрам из таблицы 1 и, тем самым, доказательства теоремы 1.1.

Во-первых, заметим, что алгебры $\mathfrak{m}_{1}, \mathfrak{m}_{4}, \mathfrak{m}_{18}$ из этого перечня являются нильпотентными. Наличие у них только вырожденных по Леви интегральных поверхностей зафиксировано в [1].

Большинство оставшихся алгебр из упомянутых 27 типов (как, впрочем, и три упомянутые нильпотентные алгебры) имеет абелевы подалгебры размерности $\geqslant 3$. Более точно, справедливы следующие утверждения.

Предложение 3.1. Тринадиать типов алгебр из таблицы 1 содержат четырехмерные абелевы идеалы.

Доказательство. Для доказательства укажем такие идеалы для десяти алгебр, не являющихся нильпотентными:

$$
\begin{aligned}
& \text { алгебра } \mathfrak{m}_{2}:\left\langle e_{1}, e_{3}, e_{4}, e_{5}\right\rangle, \\
& \text { алгебры } \mathfrak{m}_{5}, \mathfrak{m}_{6}, \mathfrak{m}_{7}, \mathfrak{m}_{8}:\left\langle e_{1}, e_{2}, e_{4}, e_{5}\right\rangle, \\
& \text { алгебры } \mathfrak{m}_{19}, \mathfrak{m}_{20}, \mathfrak{m}_{21}, \mathfrak{m}_{22}, \mathfrak{m}_{23}:\left\langle e_{1}, e_{2}, e_{3}, e_{5}\right\rangle .
\end{aligned}
$$

Предложение 3.1 доказано.

С учетом леммы 3.3 получаем следующее утверждение.

Следствие 3.1. Для 13 из 23 типов разложимых разрешимых пятимерных алгебр утверждение теоремы 1.1 верно.

Предложение 3.2. Все оставшиеся 10 типов алгебр из таблицы 1 содержат трехмерные абелевы подалгебры.

Доказательство. Достаточно непосредственно проверить следующие факты:

алгебра $\mathfrak{m}_{3}:\left\langle e_{1}, e_{3}, e_{5}\right\rangle$ - абелев идеал,

алгебры $\mathfrak{m}_{11}, \mathfrak{m}_{12}, \mathfrak{m}_{13}, \mathfrak{m}_{14}, \mathfrak{m}_{15}:\left\langle e_{1}, e_{2}, e_{4}\right\rangle$-абелев идеал,

алгебры $\mathfrak{m}_{24}, \mathfrak{m}_{25}, \mathfrak{m}_{26}:\left\langle e_{1}, e_{2}, e_{5}\right\rangle,\left\langle e_{1}, e_{3}, e_{5}\right\rangle$ - абелевы подалгебры,

алгебры $\mathfrak{m}_{27}:\left\langle e_{1}, e_{2}, e_{5}\right\rangle$ - абелев идеал.

Исследование именно этих десяти типов алгебр составляет содержание оставшейся части статьи. Доказанная выше лемма 3.2 дает возможность изучать для каждой из таких алгебр лишь два упомянутых в ней подслучая (вместо пяти, рассматривавшихся, например, в [1]).

Дополнительные упрощения рассуждений возникают при разбиении совокупности этих 10 типов на блоки, сформированные в предложении 3.2 и связанные с выделяемыми в этих блоках абелевыми идеалами и подалгебрами. 
4. Голоморфные реализации алгебры $\mathfrak{m}_{3}$. Рассмотрим алгебру $\mathfrak{m}_{3}$, определяемую коммутационными соотношениями

$$
\left[e_{1}, e_{2}\right]=e_{1}, \quad\left[e_{3}, e_{4}\right]=e_{3} .
$$

Предложение 4.1. С точностъю до голоморфной эквивалентности любая голоморфная реализация алгебры $\mathfrak{m}_{3}$ допускает невырожденные по Леви интегральные поверхности лишь из семейства

$$
v=\ln y_{1}+\alpha \ln y_{2}, \quad \alpha \in \mathbb{R} \backslash\{0\} .
$$

Доказательство. Алгебра $\mathfrak{m}_{3}$ содержит трехмерную абелеву подалгебру $\left\langle e_{1}, e_{3}, e_{5}\right\rangle$. Поэтому, основываясь на лемме 3.2 , для построения голоморфных реализаций данной алгебры будем рассматривать наборы базисных полей двух видов:

$$
\begin{aligned}
\text { Случай 1: } & e_{1}=(1,0,0), \\
e_{2} & =\left(f_{2}\left(z_{1}, z_{2}, w\right), g_{2}\left(z_{1}, z_{2}, w\right), h_{2}\left(z_{1}, z_{2}, w\right)\right), \\
e_{3} & =(0,1,0), \\
e_{4} & =\left(f_{4}\left(z_{1}, z_{2}, w\right), g_{4}\left(z_{1}, z_{2}, w\right), h_{4}\left(z_{1}, z_{2}, w\right)\right), \\
e_{5} & =(0,0,1) ; \\
\text { Случай 2: } & e_{1}=(1,0,0), \\
e_{2} & =\left(f_{2}\left(z_{1}, z_{2}, w\right), g_{2}\left(z_{1}, z_{2}, w\right), h_{2}\left(z_{1}, z_{2}, w\right)\right), \\
e_{3} & =\left(\hat{f}_{3}\left(z_{2}\right), 0, \hat{h}_{3}\left(z_{2}\right)\right), \\
& e_{4}=\left(f_{4}\left(z_{1}, z_{2}, w\right), g_{4}\left(z_{1}, z_{2}, w\right), h_{4}\left(z_{1}, z_{2}, w\right)\right), \\
& e_{5}=(0,0,1) .
\end{aligned}
$$

Рассмотрим случай 1 . Из соотношений $\left[e_{1}, e_{2}\right]=e_{1},\left[e_{2}, e_{3}\right]=0,\left[e_{2}, e_{5}\right]=0$ следует, что поле $e_{2}$ в (4.2) имеет вид

$$
e_{2}=\left(z_{1}+A_{2}, B_{2}, C_{2}\right)
$$

с некоторыми комплексными коэффициентами $A_{2}, B_{2}, C_{2}$. Аналогично, используя равенства $\left[e_{1}, e_{4}\right]=0,\left[e_{3}, e_{4}\right]=e_{3},\left[e_{4}, e_{5}\right]=0$, получаем следующий вид поля $e_{4}$ в наборе (4.2):

$$
e_{4}=\left(A_{4}, z_{2}+B_{4}, C_{4}\right) .
$$

Коммутационное соотношение $\left[e_{2}, e_{4}\right]=0$ приводит к векторному равенству

$$
\left(-A_{4}, B_{2}, 0\right)=(0,0,0),
$$

из которого следует, что $A_{4}=0$ и $B_{2}=0$.

Используя замены $z_{1}^{*}=z_{1}+A_{2}, z_{2}^{*}=z_{2}+B_{4}$ и рассматривая вместо получающихся полей $e_{2}$ и $e_{4}$ их линейные комбинации с полем $e_{5}$, приходим к окончательному виду базиса (4.2) в случае 1 (знаки «*» опускаем):

$$
\begin{aligned}
& e_{1}=(1,0,0), \\
& e_{2}=\left(z_{1}, 0, i a\right), \\
& e_{3}=(0,1,0), \\
& e_{4}=\left(0, z_{2}, i b\right), \\
& e_{5}=(0,0,1) .
\end{aligned}
$$

В записи (4.4) $a=\operatorname{Im} C_{2}, b=\operatorname{Im} C_{4}$.

Интегральная поверхность алгебры с базисом (4.4), очевидно, является трубчатой с уравнением вида $\Phi\left(y_{1}, y_{2}, v\right)=0$, которое можно записать в виде $v=F\left(y_{1}, y_{2}\right)$. Касание векторными полями $e_{2}$ и $e_{4}$ обсуждаемой интегральной поверхности эквивалентно выполнению системы двух равенств

$$
y_{1} \frac{\partial F}{\partial y_{1}}-a=0, \quad y_{2} \frac{\partial F}{\partial y_{2}}-b=0 .
$$


Решая эту систему, получаем уравнение искомой интегральной поверхности:

$$
v=a \ln y_{1}+b \ln y_{2} .
$$

Так как нас интересуют только невырожденные поверхности, то в нем $a \neq 0, b \neq 0$. Тогда растяжением переменной $w^{*}=w / a$ это уравнение сводится к (4.1).

Перейдем теперь к рассмотрению случая 2, то есть набора базисных полей (4.3). Используя соотношения $\left[e_{1}, e_{4}\right]=0,\left[e_{4}, e_{5}\right]=0$, получаем, что функции $f_{4}, g_{4}, h_{4}$ не зависят от переменных $z_{1}$ и $w$. Таким образом,

$$
e_{4}=\left(\hat{f}_{4}\left(z_{2}\right), \hat{g}_{4}\left(z_{2}\right), \hat{h}_{4}\left(z_{2}\right)\right)
$$

Если допустить, что $\hat{g}_{4}\left(z_{2}\right)$ тождественно обращается в нуль, то в базисе (4.3) окажется четыре поля с тождественно нулевыми вторыми компонентами, что, согласно лемме 3.4, приводит к вырождению по Леви соответствующей интегральной поверхности. Поэтому можем считать, что (возможно, после сдвига начала координат) $\hat{g}_{4}(0) \neq 0$. Следовательно, пользуясь замечанием 3.2 , поле $e_{4}$ можно привести к виду $e_{4}=(0,1,0)$. Тогда из соотношений

$$
\left[e_{1}, e_{2}\right]=e_{1}, \quad\left[e_{2}, e_{4}\right]=0, \quad\left[e_{2}, e_{5}\right]=0
$$

следует, что поле $e_{2}$ в (4.3) имеет вид $e_{2}=\left(z_{1}+A_{2}, B_{2}, C_{2}\right)$. Используя равенство $\left[e_{3}, e_{4}\right]=e_{3}$, получаем следующее соотношение:

$$
\left(-\hat{f}_{3}^{\prime}\left(z_{2}\right), 0,-\hat{h}_{3}^{\prime}\left(z_{2}\right)\right)=\left(\hat{f}_{3}\left(z_{2}\right), 0, \hat{h}_{3}\left(z_{2}\right)\right),
$$

откуда $\hat{f}_{3}=A_{3} e^{-z_{2}}, \hat{h}_{3}=C_{3} e^{-z_{2}}$. Последнее неиспользованное коммутационное соотношение $\left[e_{2}, e_{3}\right]=0$ приводит к векторному равенству

$$
\left(-A_{3}\left(B_{2}+1\right) e^{-z_{2}}, 0,-B_{2} C_{3} e^{-z_{2}}\right)=(0,0,0) .
$$

Таким образом, должна выполняться система уравнений

$$
A_{3}\left(B_{2}+1\right)=0, \quad B_{2} C_{3}=0 .
$$

Ее решениями являются три набора:

$$
\text { (1) } B_{2}=0, A_{3}=0 ; \quad \text { (2) } A_{3}=0, C_{3}=0 ; \quad \text { (3) } B_{2}=-1, C_{3}=0 .
$$

Очевидно, что при подстановке любого из этих трех наборов в пятерку

$$
\begin{aligned}
& e_{1}=(1,0,0), \\
& e_{2}=\left(z_{1}+A_{2}, B_{2}, C_{2}\right), \\
& e_{3}=\left(A_{3} e^{-z_{2}}, 0, C_{3} e^{-z_{2}}\right), \\
& e_{4}=(0,1,0), \\
& e_{5}=(0,0,1),
\end{aligned}
$$

в ней всегда найдется пара полей, гарантирующая либо вырождение по Леви интегральной поверхности, либо ранг алгебры, меньший пяти. Предложение 4.1 доказано.

5. Голоморфные реализации алгебр $\mathfrak{m}_{11}-\mathfrak{m}_{15}$. Содержание этого раздела посвящено доказательству следующего утверждения.

Теорема 5.1. Любая невырожденная по Леви однородная гиперповерхность, ассочиированная с одной из алгебр типов $\mathfrak{m}_{11}-\mathfrak{m}_{15}$, либо является сферической индефинитной поверхностью, либо голоморфно эквивалентна одной из трубчатых поверхностей
(i) $v=y_{1}^{2}+\varepsilon \ln y_{2} \quad(\varepsilon= \pm 1)$
или
(ii) $v \sin y_{2}+y_{1} \cos y_{2}=e^{p y_{2}} \quad(p \in \mathbb{R})$.

Замечание 5.1. Поверхности из теоремы 5.1 являются известными трубками. При этом поверхности из п. (i) имеют аффинно-однородные основания и семимерные алгебры симметрий, выписанные в [21]. Семейство из п. (ii) также имеется в [21], но «максимальные» алгебры симметрий для них имеют размерность 6 , а основания этих трубок не являются аффинно-однородными. 
Доказательные обсуждения теоремы 5.1 начнем с напоминания о наличии у каждой алгебры из обсуждаемого списка абелева идеала $\left\langle e_{1}, e_{2}, e_{4}\right\rangle$. Ссылаясь на лемму 3.2 , изучение голоморфных реализаций этих алгебр можно свести к двум случаям, связанным с упрощением именно тройки полей $e_{1}, e_{2}, e_{4}$.

Отметим также, что абелевой подалгеброй (но не идеалом) любой из пяти алгебр $\mathfrak{m}_{11}-\mathfrak{m}_{15}$ является линейная оболочка другой тройки базисных полей $\mathfrak{m}_{11}-\mathfrak{m}_{15}$, а именно, $\left\langle e_{1}, e_{2}, e_{5}\right\rangle$. Это замечание также будет использовано в дальнейшем, но начнем мы с тройки полей $e_{1}, e_{2}, e_{4}$.

Первый случай леммы 3.2. В этом случае тройку $e_{1}, e_{2}, e_{4}$ можно считать (для любой из пяти алгебр $\left.\mathfrak{m}_{11}-\mathfrak{m}_{15}\right)$ выпрямленной и имеющей вид

$$
e_{1}=(1,0,0), \quad e_{2}=(0,1,0), \quad e_{4}=(0,0,1) .
$$

Для рассмотрения в едином ключе всех пяти алгебр перейдем к универсальной форме имеющихся в них коммутационных соотношений. Несложно убедиться, что для любой из этих алгебр содержательные соотношения можно описать тремя формулами

$$
\left[e_{1}, e_{3}\right]=\alpha e_{1}+\beta e_{2}, \quad\left[e_{2}, e_{3}\right]=\lambda e_{1}+\mu e_{2}, \quad\left[e_{4}, e_{5}\right]=e_{4} .
$$

Наборы вещественных коэффициентов $\alpha, \beta, \lambda, \mu$ для всех пяти типов алгебр описываются при этом следующей таблицей:

\begin{tabular}{l|rrrr} 
& $\alpha$ & $\beta$ & $\lambda$ & $\mu$ \\
\hline $\mathfrak{m}_{11}$ & 0 & 0 & 1 & 0 \\
$\mathfrak{m}_{12}$ & 1 & 0 & 1 & 1 \\
$\mathfrak{m}_{13}$ & 1 & 0 & 0 & 1 \\
$\mathfrak{m}_{14}$ & 1 & 0 & 0 & $s$ \\
$\mathfrak{m}_{15}$ & $p$ & -1 & 1 & $p$
\end{tabular}

Предложение 5.1. Если базис пятимерной алгебры $g$ голоморбных векторных полей в $\mathbb{C}^{3}$ удовлетворяет условиям (5.1), а отличные от (5.2) коммутационные соотношения в $g$ являются тривиальными, то базис g имеет вид

$$
\begin{aligned}
& e_{1}=(1,0,0), \\
& e_{2}=(0,1,0), \\
& e_{3}=\left(\alpha z_{1}+\lambda z_{2}+A_{3}, \beta z_{1}+\mu z_{2}+B_{3}, 0\right), \\
& e_{4}=(0,0,1), \\
& e_{5}=\left(A_{5}, B_{5}, w+C_{5}\right)
\end{aligned}
$$

с некоторыми комплексными постолнными $A_{k}, B_{k}, C_{k}$. При этом выполняются два дополнительных соотношения

$$
\alpha A_{5}+\lambda B_{5}=0, \quad \beta A_{5}+\mu B_{5}=0 .
$$

Доказательство. Три тривиальных коммутационных соотношения (из десяти, имеющихся в произвольной пятимерной алгебре) использованы нами для выпрямления базисной тройки полей $e_{1}$, $e_{2}, e_{4}$. Для уточнения вида поля $e_{5}$ рассмотрим еще три соотношения

$$
\left[e_{1}, e_{5}\right]=0, \quad\left[e_{2}, e_{5}\right]=0, \quad\left[e_{4}, e_{5}\right]=e_{4} .
$$

Два первых из них означают, что компоненты поля

$$
e_{5}=\left(f_{5}\left(z_{1}, z_{2}, w\right), g_{5}\left(z_{1}, z_{2}, w\right), h_{5}\left(z_{1}, z_{2}, w\right)\right)
$$

не зависят от переменных $z_{1}, z_{2}$. Третье соотношение можно записать в виде векторного равенства

$$
\left(\frac{\partial f_{5}}{\partial w}, \frac{\partial g_{5}}{\partial w}, \frac{\partial h_{5}}{\partial w}\right)=(0,0,1) .
$$

Ясно, что из него следует заявленный в предложении 5.1 вид поля $e_{5}$. 
Аналогичное рассмотрение трех коммутационных соотношений, содержащих поле $e_{3}$, т.е. $\left[e_{3}, e_{4}\right]=0$, а также двух первых соотношений из набора (5.2), приводят к пошаговому упрощению вида этого поля.

Так, из соотношения $\left[e_{3}, e_{4}\right]=0$ получаем первое упрощение:

$$
e_{3}=\left(f_{3}\left(z_{1}, z_{2}\right), g_{3}\left(z_{1}, z_{2}\right), h_{3}\left(z_{1}, z_{2}\right)\right) ;
$$

затем из соотношения $\left[e_{1}, e_{3}\right]=\alpha e_{1}+\beta e_{2}-$ второе:

$$
e_{3}=\left(\alpha z_{1}+\hat{f}_{3}\left(z_{2}\right), \beta z_{1}+\hat{g}_{3}\left(z_{2}\right), \hat{h}_{3}\left(z_{2}\right)\right) ;
$$

и, наконец, из соотношения $\left[e_{2}, e_{3}\right]=\lambda e_{1}+\mu e_{2}$ - третье:

$$
e_{3}=\left(\alpha z_{1}+\lambda z_{2}+A_{3}, \beta z_{1}+\mu z_{2}+B_{3}, C_{3}\right) .
$$

Для завершения доказательства предложения 5.1 рассмотрим коммутатор $\left[e_{3}, e_{5}\right]=0$ для упрощенных полей $e_{3}$ и $e_{5}$. Здесь возникает векторное равенство

$$
C_{3}(0,0,1)-A_{5}(\alpha, \beta, 0)-B_{5}(\lambda, \mu, 0)=(0,0,0) .
$$

Из рассмотрения его отдельных компонент вытекают равенство $C_{3}=0$ и соотношения (5.4). Предложение 5.1 доказано.

Предложение 5.2. Интегральная поверхность любой алгебры векторных полей с базисом вида (5.3)-(5.4) является трубкой над аффинно-однородным основанием.

Доказательство. Определяющая функция $\Phi\left(z_{1}, z_{2}, w\right)$ интегральной поверхности

$$
M=\left\{\Phi\left(z_{1}, z_{2}, w\right)=0\right\}
$$

произвольной пятимерной алгебры $g$ голоморфных векторных полей с базисом $e_{1}, e_{2}, e_{3}, e_{4}, e_{5}$ удовлетворяет общеизвестным условиям касания

$$
\operatorname{Re}\left(\left.e_{k}(\Phi)\right|_{M}\right) \equiv 0, \quad k=1, \ldots, 5 .
$$

В случае базиса (5.3), содержащего три тривиальных дифференцирования $\partial / \partial z_{1}, \partial / \partial z_{2}, \partial / \partial w$, условия (5.5) означают независимость функции $\Phi\left(z_{1}, z_{2}, w\right)$ от трех вещественных переменных $x_{1}=\operatorname{Re} z_{1}, x_{2}=\operatorname{Re} z_{2}, u=\operatorname{Re} w$. Другими словами, поверхность $M$ является трубкой над поверхностью

$$
\Gamma=\left\{\Phi\left(y_{1}, y_{2}, v\right)=0\right\}
$$

из вещественного трехмерного пространства с координатами $y_{1}, y_{2}, v$. С учетом этого два оставшихся соотношения (5.5) превращаются в систему двух уравнений в частных производных

$$
\begin{gathered}
\operatorname{Re}\left(\left(\alpha y_{1}+\lambda y_{2}+\operatorname{Im} A_{3}\right) \frac{\partial \Phi}{\partial y_{1}}+\left(\beta y_{1}+\mu y_{2}+\operatorname{Im} B_{3}\right) \frac{\partial \Phi}{\partial y_{2}}\right)=0, \\
\operatorname{Re}\left(\left(\operatorname{Im} A_{5}\right) \frac{\partial \Phi}{\partial y_{1}}+\left(\operatorname{Im} B_{5}\right) \frac{\partial \Phi}{\partial y_{2}}+\left(v+\operatorname{Im} C_{5}\right) \frac{\partial \Phi}{\partial v}\right)=0 .
\end{gathered}
$$

Эта система означает, что поверхность (5.6) является аффинно-однородной интегральной поверхностью для (абелевой) алгебры аффинных векторных полей с базисом

$$
\begin{gathered}
E_{3}=\left(\alpha y_{1}+\lambda y_{2}+\operatorname{Im} A_{3}\right) \frac{\partial}{\partial y_{1}}+\left(\beta y_{1}+\mu y_{2}+\operatorname{Im} B_{3}\right) \frac{\partial}{\partial y_{2}}, \\
E_{5}=\left(\operatorname{Im} A_{5}\right) \frac{\partial}{\partial y_{1}}+\left(\operatorname{Im} B_{5}\right) \frac{\partial}{\partial y_{2}}+\left(v+\operatorname{Im} C_{5}\right) \frac{\partial}{\partial v} .
\end{gathered}
$$

Предложение 5.2 доказано.

Уточним полученное общее утверждение за счет более подробного рассмотрения алгебр $\mathfrak{m}_{11^{-}}$ $\mathfrak{m}_{15}$ в обсуждаемом первом случае. 
Предложение 5.3. Всякая однородная невырожденная поверхность, ассочиированная в первом случае с алгеброй $\mathfrak{m}_{11}$, голоморфно эквивалентна одной из поверхностей семейства

$$
v=y_{1}^{2}+\varepsilon \ln y_{2} \quad(\varepsilon= \pm 1) ;
$$

алгебрам $\mathfrak{m}_{12}-\mathfrak{m}_{15}$ соответствуют в первом случае лишь вырожденные по Леви поверхности.

Доказательство. Рассмотрим сначала соотношения (5.4) для алгебр $\mathfrak{m}_{12}-\mathfrak{m}_{15}$. С учетом таблицы значений коэффициентов $\alpha, \beta, \lambda, \mu$ для всех этих четырех типов алгебр получаем равенства $A_{5}=B_{5}=0$. В таком случае наличие в любой из этих алгебр полей $e_{5}=\left(0,0, w+C_{5}\right)$ и $e_{4}=(0,0,1)$ означает вырожденность по Леви любой интегральной поверхности таких алгебр.

Теперь обсудим алгебру $\mathfrak{m}_{11}$. Для нее с учетом соотношений (5.4) получаем поля $e_{3}, e_{5}$ в виде

$$
e_{3}=\left(z_{2}+A_{3}, B_{3}, 0\right), \quad e_{5}=\left(A_{5}, 0, w+C_{5}\right) .
$$

Заменяя эти поля их комбинациями с полями из выпрямленной тройки $e_{1}, e_{2}, e_{4}$, можно считать, что все константы в формулах (5.8) - чисто мнимые. Система дифференциальных уравнений в частных производных, соответствующая двум этим полям и уравнениям $v=F\left(y_{1}, y_{2}\right)$ искомых однородных поверхностей, имеет вид

$$
\left(y_{2}+a_{3}\right) \frac{\partial F}{\partial y_{1}}+b_{3} \frac{\partial F}{\partial y_{2}}=0, \quad a_{5} \frac{\partial F}{\partial y_{1}}=F+c_{5} .
$$

Все константы в этих уравнениях являются мнимыми частями одноименных констант из формул (5.8), обозначенных заглавными буквами.

Отметим, что при $a_{5}=0$ или $b_{3}=0$ решение системы (5.9) очевидно приводит к вырожденным поверхностям.

При ненулевых коэффициентах $a_{5}, b_{3}$ сначала получаем решение второго уравнения в виде

$$
F=-c_{5}+H\left(y_{2}\right) e^{y_{1} / a_{5}}
$$

с произвольной аналитической функцией $H\left(y_{2}\right)$. Тогда первое уравнение $(5.9)$ примет вид (после деления обеих его частей на $\left.e^{y_{1} / a_{5}}\right)$

$$
H^{\prime}\left(y_{2}\right)=N\left(y_{2}+a_{3}\right) H\left(y_{2}\right), \quad N=-\frac{1}{a_{5} b_{3}} .
$$

Отсюда получаем общее решение системы (5.9) в виде

$$
F=-c_{5}+D \exp \left(\frac{N}{2}\left(y_{2}+a_{3}\right)^{2}\right) e^{y_{1} / a_{5}}
$$

с произвольной константой $D$. Логарифмируя теперь уравнение $v=F$ (при положительном $D$ ), получим его вариант

$$
\ln \left(v+c_{5}\right)=\frac{N}{2}\left(y_{2}+a_{3}\right)^{2}+\frac{1}{a_{5}} y_{1}+\ln D,
$$

который сводится к (5.7) несложными аффинными преобразованиями. Предложение 5.3 доказано.

Второй случай леммы 3.2. Будем рассматривать базис любой из пяти алгебр $\mathfrak{m}_{11}-\mathfrak{m}_{15}$, считая, что тройка элементов $e_{1}, e_{2}, e_{4}$ этого базиса упрощена до состояния

$$
e_{1}=(1,0,0), \quad e_{2}=(0,0,1), \quad e_{4}=\left(\hat{f}_{4}, 0, \hat{h}_{4}\right) .
$$

Замечание 5.2. Здесь мы выпрямляем поля и связываем их с координатными дифференцированиями в порядке, отличном от обсуждений предыдущего раздела. На окончательный итог обсуждений такие перестановки, разумеется, не влияют, но некоторые фрагменты рассуждений упрощаются за счет таких перестановок. 
Напомним, что поле $e_{5}$ коммутирует с полями $e_{1}, e_{2}$. Это означает, что при выпрямленных $e_{1}$, $e_{2}$ поле $e_{5}$ имеет следующий упрощенный вид:

$$
e_{5}=\left(\hat{f}_{5}\left(z_{2}\right), \hat{g}_{5}\left(z_{2}\right), \hat{h}_{5}\left(z_{2}\right)\right) .
$$

При этом в силу леммы 3.4 для четверки базисных (линейно независимых над $\mathbb{R} !)$ полей

$$
\begin{aligned}
e_{1} & =(0,0,1), \\
c & e_{2}=(1,0,0), \\
e_{4} & =\left(\hat{f}_{4}, 0, \hat{h}_{4}\right), \\
e_{5} & =\left(\hat{f}_{5}\left(z_{2}\right), \hat{g}_{5}\left(z_{2}\right), \hat{h}_{5}\left(z_{2}\right)\right)
\end{aligned}
$$

на невырожденной по Леви поверхности невозможно тождественное равенство нулю столбца из их вторых компонент. Осуществим (если необходимо) малый сдвиг в точку на поверхности, в которой $\hat{g}_{5} \neq 0$. Далее, пользуясь замечанием 3.2 , выпрямим поле $e_{5}$ до состояния $(0,1,0)$. При этом вид выпрямленных ранее полей $e_{1}, e_{2}$ также сохранится.

После этого мы имеем базис голоморфной реализации любой из пяти алгебр $\mathfrak{m}_{11}-\mathfrak{m}_{15}$ в виде

$$
\begin{aligned}
& e_{1}=(0,0,1), \\
& e_{2}=(1,0,0), \\
& e_{3}=\left(f_{3}\left(z_{1}, z_{2}, w\right), g_{3}\left(z_{1}, z_{2}, w\right), h_{3}\left(z_{1}, z_{2}, w\right)\right), \\
& e_{4}=\left(\hat{f}_{4}, 0, \hat{h}_{4}\right), \\
& e_{5}=(0,1,0) .
\end{aligned}
$$

Невырожденные по Леви интегральные поверхности (если они существуют) любой алгебры с таким базисом являются трубчатыми в связи с наличием в алгебре трех дифференцирований по отдельным координатам. При этом имеют место два следующих утверждения.

Предложение 5.4. Если алгебра g голоморфных векторных полей в пространстве $\mathbb{C}^{3}$ имеет базис вида (5.11) и структуру одной из четырех алгебр $\mathfrak{m}_{11}-\mathfrak{m}_{14}$, то всякая интегральная поверхность такой алгебры является вырожденной по Леви.

Предложение 5.5. Пусть алгебра g голоморфных векторных полей в пространстве $\mathbb{C}^{3}$ имеет базис вида (5.11) и структуру $\mathfrak{m}_{15}$, а $M$ - интегральная поверхность такой алгебры. Тогда $M$ либо является вырожденной по Леви, либо голоморфно эквивалентна одной из следующих аффинно-однородных индефинитных поверхностей:

$$
\begin{aligned}
& v=\arg \left(y_{1}+i y_{2}\right), \\
& y_{1} x_{2}-v y_{2}=\left|z_{2}\right| e^{B \arg z_{2}}, \quad B \in \mathbb{R} .
\end{aligned}
$$

Замечание 5.3. Поверхность (5.12) голоморфно эквивалентна сферической (см. [6]) поверхности $v=y_{1} \operatorname{tg} y_{2}$.

Замечание 5.4. Поверхности из семейства (5.13) не являются трубчатыми. В то же время любая из них голоморфно эквивалентна одной из трубок

$$
v \cos y_{2}+y_{1} \sin y_{2}=e^{p y_{2}}, \quad p \geqslant 0 .
$$

Замечание 5.5. Основание трубчатой поверхности (5.14), описываемое этим же уравнением в пространстве трех вещественных переменных $y_{1}, y_{2}, v$ не является аффинно-однородной поверхностью. В работе [21], посвященной изучению голоморфно-однородных поверхностей с богатыми алгебрами симметрии, построено несколько семейств таких «странных» трубок и, в частности, семейство (5.14).

Доказательство предложсния 5.4. Изучим еще не рассмотренные коммутационные соотношения. Например, из равенства $\left[e_{4}, e_{5}\right]=0$ для полей вида (5.11) получаем уточненный вид поля

$$
e_{4}=e^{-z_{2}}\left(A_{4}, 0, C_{4}\right)
$$


с некоторыми комплексными постоянными. Далее, равенство $\left[e_{3}, e_{5}\right]=0$ означает, что компоненты поля $e_{3}$ не зависят от переменной $z_{2}$. Из рассмотрения коммутаторов

$$
\left[e_{1}, e_{3}\right]=\alpha e_{1}+\beta e_{2}, \quad\left[e_{2}, e_{3}\right]=\lambda e_{1}+\mu e_{2}
$$

получаем следующий вид поля $e_{3}$ :

$$
e_{3}=\left(\beta w+\mu z_{1}+A_{3}, B_{3}, \alpha w+\lambda z_{1}+C_{3}\right)
$$

с некоторыми (комплексными) константами $A_{3}, B_{3}, C_{3}$.

Отметим, что для алгебр, допускающих невырожденные интегральные поверхности, коэффициент $B_{3} \neq 0$ по аналогии с уже обсуждавшейся выше четверкой полей (5.10).

Остается рассмотреть последнее коммутационное соотношение $\left[e_{3}, e_{4}\right]=0$ с учетом полученной информации о полях $e_{3}, e_{4}$. Из него получаем векторное равенство

$$
-B_{3} e^{-z_{2}}\left(A_{4}, 0, C_{4}\right)-e^{-z_{2}}\left(A_{4}(\mu, 0, \lambda)+C_{4}(\beta, 0, \alpha)\right)=(0,0,0) .
$$

Две компоненты этого равенства являются содержательными и имеют вид

$$
A_{4}\left(B_{3}+\mu\right)+C_{4} \beta=0, \quad A_{4} \lambda+C_{4}\left(B_{3}+\alpha\right)=0 .
$$

Проверим совместность этих условий с видом полученных базисов

$$
\begin{aligned}
& e_{1}=(0,0,1), \\
& e_{2}=(1,0,0), \\
& e_{3}=\left(\beta w+\mu z_{1}+A_{3}, B_{3}, \alpha w+\lambda z_{1}+C_{3}\right), \\
& e_{4}=\left(A_{4} e^{-z_{2}}, 0, C_{4} e^{-z_{2}}\right), \\
& e_{5}=(0,1,0)
\end{aligned}
$$

обсуждаемых алгебр и с требованием невырожденности интегральных поверхностей таких алгебр.

I. Для алгебры $\mathfrak{m}_{11}$ с набором $(\alpha, \beta, \lambda, \mu)=(0,0,1,0)$ система уравнений (5.15) превращается в систему

$$
A_{4} B_{3}=0, \quad A_{4}+C_{4} B_{3}=0 .
$$

При $B_{3} \neq 0$ из нее следуют равенства $A_{4}=C_{4}=0$, невозможные для базисного поля $e_{4}$ обсуждаемой алгебры.

II. Для алгебры $\mathfrak{m}_{12}$ с набором $(\alpha, \beta, \lambda, \mu)=(1,0,1,1)$ система двух уравнений (5.15) превращается в систему

$$
A_{4}\left(B_{3}+1\right)=0, \quad A_{4}+C_{4}\left(B_{3}+1\right)=0 .
$$

Заметим, что для любого решения этой системы выполняется равенство $A_{4}=0$. В то же время при таком условии в алгебре с базисом (5.16) мы имеем два комплексно пропорциональных поля $e_{1}$ и $e_{4}$. Это приводит к вырождению по Леви любой интегральной поверхности такой алгебры.

III. Для алгебры $\mathfrak{m}_{13}$ с набором $(\alpha, \beta, \lambda, \mu)=(1,0,0,1)$ система двух уравнений (5.15) превращается в систему

$$
A_{4}\left(B_{3}+1\right)=0, \quad C_{4}\left(B_{3}+1\right)=0 .
$$

При $B_{3} \neq-1$ из этой системы следует одновременное обращение в нуль коэффициентов $A_{3}, B_{3}$, что невозможно для базисного поля $e_{4}$, как отмечалось выше в случае $\mathfrak{m}_{11}$. При $B_{3}=-1$ четверка линейно независимых над $\mathbb{R}$ полей $e_{1}, e_{2}, e_{3}+e_{5}, e_{4}$ из базиса (5.16) имеет тождественно нулевые вторые компоненты. Как и при рассмотрении базиса (5.10), это несовместимо с невырожденностью по Леви интегральных поверхностей такой алгебры.

IV. Для алгебры $\mathfrak{m}_{14}$ с набором $(\alpha, \beta, \lambda, \mu)=(1,0,0, s)$ система двух уравнений (5.15) превращается в систему

$$
A_{4}\left(B_{3}+s\right)=0, \quad C_{4}\left(B_{3}+1\right)=0 .
$$

Напомним, что здесь выполняется неравенство $s \neq 1$ для параметра $s$. Тогда из (5.17) следует, что либо $A_{4}=0$, либо $C_{4}=0$. В каждом из этих случаев в базисе $(5.16)$ найдется пара комплексно 
пропорциональных полей ( $e_{1}$ и $e_{4}$ или $e_{2}$ и $\left.e_{4}\right)$. Как и в предыдущем случае, это ведет к вырождению по Леви любой интегральной поверхности соответствующей алгебры. Предложение 5.4 доказано.

Доказательство предложения 5.5. Для алгебры $\mathfrak{m}_{15}$ с набором $(\alpha, \beta, \lambda, \mu)=(p,-1,1, p)$ получаем систему (5.15) в виде

$$
A_{4}\left(B_{3}+p\right)-C_{4}=0, \quad A_{4}+C_{4}\left(B_{3}+p\right)=0 .
$$

При каждом фиксированном $p$ имеется ровно две возможности для выполнения этих равенств с ненулевыми значениями $A_{4}, C_{4}$. Обе эти возможности связаны с равенством $\left(B_{3}+p\right)^{2}=-1$ и описываются в итоге соотношениями

$$
B_{3}=-p+\varepsilon i \quad(\varepsilon= \pm 1) .
$$

Базисы соответствующих алгебр имеют в этих ситуациях вид

$$
\begin{aligned}
& e_{1}=(0,0,1), \\
& e_{2}=(1,0,0), \\
& e_{3}=\left(-w+p z_{1}+A_{3},-p+i \varepsilon, p w+z_{1}+C_{3}\right), \\
& e_{4}=\left(A_{4} e^{-z_{2}}, 0, C_{4} e^{-z_{2}}\right), \\
& e_{5}=(0,1,0) .
\end{aligned}
$$

Такой набор полей допускает еще несколько простых преобразований. Например, сдвиги переменных

$$
z_{1}^{*}=z_{1}+\frac{A_{3} p-C_{3}}{1+p^{2}}, \quad w^{*}=w+\frac{C_{3} p-A_{3}}{1+p^{2}}
$$

освобождают формулы (5.20) от констант $A_{3}, C_{3}$. Рассмотрение вместо поля $e_{3}$ комбинации $e_{3}+$ $p e_{5}$ еще более упрощает формулу для $e_{3}$ и приводит ее к виду

$$
e_{3}=\left(-w+p z_{1}, \varepsilon i, p w+z_{1}\right) .
$$

Поскольку из (5.18) и (5.19) следует, что $C_{4}=i \varepsilon A_{4}$, и для базисного поля $e_{4}$ коэффициенты $C_{4}$, $A_{4}$ не могут быть нулевыми, то растяжением можно привести это поле к виду

$$
e_{4}=\left(e^{i t} e^{-z_{2}}, 0, i \varepsilon e^{i t} e^{-z_{2}}\right)=\left(e^{i t-z_{2}}, 0, i \varepsilon e^{i t-z_{2}}\right) .
$$

Еще одним сдвигом $z_{2}^{*}=z_{2}-i t$ приводим базисы обсуждаемых алгебр к виду

$$
\begin{aligned}
& e_{1}=(0,0,1), \\
& e_{2}=(1,0,0), \\
& e_{3}=\left(-w+p z_{1}, i \varepsilon, p w+z_{1}\right), \\
& e_{4}=\left(e^{-z_{2}}, 0, i \varepsilon e^{-z_{2}}\right), \\
& e_{5}=(0,1,0) .
\end{aligned}
$$

Интегральная поверхность $M$ любой такой алгебры, очевидно, является трубчатой поверхностью и может быть задана уравнением $v=F\left(y_{1}, y_{2}\right)$. Непосредственное интегрирование алгебры (5.21) сводится к решению системы двух уравнений в частных производных

$$
\left(-F+p y_{1}\right) \frac{\partial F}{\partial y_{1}}+\varepsilon \frac{\partial F}{\partial y_{1}}=\left(p F+y_{1}\right), \quad-e^{-x_{2}} \sin y_{2} \frac{\partial F}{\partial y_{1}}=\varepsilon e^{-x_{2}} \cos y_{2}
$$

относительно функции $F\left(y_{1}, y_{2}\right)$. Решение системы (5.22) имеет вид

$$
F=\varepsilon y_{1} \frac{\cos y_{2}}{\sin y_{2}}+\frac{C}{\sin y_{2}} e^{\varepsilon p y_{2}}
$$

где $C$ - произвольная вещественная константа. Соответствующие уравнения однородных поверхностей (после очевидных упрощений) можно записать в виде

$$
v \sin y_{2}+y_{1} \cos y_{2}=C e^{\varepsilon p y_{2}} .
$$

При $C=0$ уравнение поверхности (5.23) простыми переобозначениями переменных превращается в уравнение известной (см., например, [20]) трубчатой поверхности (5.12) с аффинно-однородным 
основанием. При ненулевых $C$ растяжение переменных $z_{1}=\varepsilon C z_{1}^{*}, z_{2}=\varepsilon z_{2}^{*}, w=C w^{*}$ приводит уравнение (5.23) к виду (5.13), т.е.

$$
v \sin y_{2}+y_{1} \cos y_{2}=e^{p y_{2}} .
$$

Завершая доказательство предложения 5.5, заметим, что при голоморфной замене координат $z_{2}^{*}=e^{-z_{2}}$ поля базиса (5.21) приобретают вид

$$
\begin{aligned}
& e_{1}=(0,0,1), \\
& e_{2}=(1,0,0), \\
& e_{3}=\left(-w+p z_{1},-i \varepsilon z_{2}, p w+z_{1}\right), \\
& e_{4}=\left(z_{2}, 0, i \varepsilon z_{2}\right), \\
& e_{5}=\left(0,-z_{2}, 0\right)
\end{aligned}
$$

и все они становятся аффинными векторными полями. Это означает, что интегральные поверхности такой аффинной алгебры являются аффинно-однородными. Уравнения таких поверхностей (приведенные без подробного вывода) имеют вид

$$
y_{1} x_{2}-v y_{2}=\left|z_{2}\right| e^{B \arg z_{2}}, \quad B \in \mathbb{R} .
$$

При этом в отличие от трубок над аффинно-однородными вещественными основаниями, коэффициенты аффинных отображений, преобразующих поверхности (5.24) в себя, являются, вообще говоря, комплексными, а не вещественными. Предложение 5.5 доказано.

6. Голоморфные реализации алгебр $\mathfrak{m}_{24}-\mathfrak{m}_{26}$. Сформулируем основное утверждение этого раздела.

Теорема 6.1. Любая невырожденная по Леви однородная гиперповерхность, ассочиированная с одной из алгебр типов $\mathfrak{m}_{24}-\mathfrak{m}_{26}$, либо является сферической поверхностью, либо голоморфно эквивалентна трубчатой поверхности

$$
v=y_{1} y_{2}+y_{2} \ln y_{2} .
$$

Доказательство этой теоремы проводится по описанной выше схеме и начинается (общим образом для всех трех алгебр) с упрощения базиса абелевой подалгебры $\left\langle e_{1}, e_{3}, e_{5}\right\rangle$. Обсуждения любого из этих трех типов алгебр начинаем с выпрямления (по лемме 3.2 ) двух полей $e_{1}, e_{3}$ и рассмотрения после этого двух случаев, связанных со свойствами поля $e_{5}$.

В первом случае леммы 3.2 для всех трех алгебр будем обсуждать пятерку базисных полей вида

$$
\begin{aligned}
& e_{1}=(0,0,1), \\
& e_{3}=(1,0,0), \\
& e_{5}=(0,1,0), \\
& e_{2}=\left(f_{2}, g_{2}, h_{2}\right), \\
& e_{4}=\left(f_{4}, g_{4}, h_{4}\right)
\end{aligned}
$$

с некоторыми голоморфными функциями $f_{k}, g_{k}, h_{k}(k=2,4)$.

Схема уточнения свойств этих функций связана с коммутационными соотношениями, имеющимися в этих алгебрах, и является общей как для первого, так и для второго случаев. Два первых шага этой схемы связаны с рассмотрением тройки («универсально устроенных») коммутаторов

$$
\left[e_{1}, e_{2}\right]=0, \quad\left[e_{2}, e_{3}\right]=e_{1}, \quad\left[e_{2}, e_{5}\right]=0,
$$

получаемых с участием поля $e_{2}$ (первый шаг), и аналогичной тройки, связанной с полем $e_{4}$ (второй шаг). На втором шаге три алгебры $\mathfrak{m}_{24}, \mathfrak{m}_{25}, \mathfrak{m}_{26}$ имеют заметные различия:

$$
\begin{array}{ll}
\mathfrak{m}_{24}: & {\left[e_{1}, e_{4}\right]=2 e_{1}, \quad\left[e_{3}, e_{4}\right]=e_{2}+e_{3}, \quad\left[e_{4}, e_{5}\right]=0,} \\
\mathfrak{m}_{25}: & {\left[e_{1}, e_{4}\right]=(1+q) e_{1}, \quad\left[e_{3}, e_{4}\right]=q e_{3}, \quad\left[e_{4}, e_{5}\right]=0 \quad(|q| \leqslant 1),} \\
\mathfrak{m}_{26}: & {\left[e_{1}, e_{4}\right]=2 p e_{1}, \quad\left[e_{3}, e_{4}\right]=e_{2}+p e_{3}, \quad\left[e_{4}, e_{5}\right]=0 \quad(p \geqslant 0) .}
\end{array}
$$


Завершающим шагом общей схемы является рассмотрение последнего коммутационного соотношения, связывающего поля $e_{2}$ и $e_{4}$ :

$$
\begin{aligned}
& \mathfrak{m}_{24}, \mathfrak{m}_{25}:\left[e_{2}, e_{4}\right]=e_{2}, \\
& \mathfrak{m}_{26}:\left[e_{2}, e_{4}\right]=p e_{2}-e_{3} .
\end{aligned}
$$

Первый шаг для всех трех алгебр обсудим общим образом. Здесь нулевые коммутаторы из тройки (6.1) означают, что компоненты $f_{2}, g_{2}, h_{2}$ поля $e_{2}$ не зависят от переменных $z_{2}, w$. Третье равенство $\left[e_{2}, e_{3}\right]=e_{1}$ из (6.1) приводит к формуле

$$
e_{2}=\left(A_{2}, B_{2},-z_{1}+C_{2}\right)
$$

с некоторыми комплексными константами $A_{2}, B_{2}, C_{2}$.

В итоге, различия в обсуждении трех алгебр в случае 1 леммы 3.2 начинаются с базисов, имеющих вид

$$
\begin{aligned}
& e_{1}=(0,0,1), \\
& e_{3}=(1,0,0), \\
& e_{5}=(0,1,0), \\
& e_{2}=\left(A_{2}, B_{2},-z_{1}+C_{2}\right), \\
& e_{4}=\left(f_{4}, g_{4}, h_{4}\right) .
\end{aligned}
$$

6.1. Алгебра $\mathfrak{m}_{24}$. Коммутационные соотношения в алгебре $\mathfrak{m}_{24}$ имеют вид

$$
\left[e_{2}, e_{3}\right]=e_{1}, \quad\left[e_{1}, e_{4}\right]=2 e_{1}, \quad\left[e_{2}, e_{4}\right]=e_{2}, \quad\left[e_{3}, e_{4}\right]=e_{2}+e_{3} .
$$

Предложение 6.1. Любая голоморфная реализация алгебры $\mathfrak{m}_{24}$ допускает лишь вырожденные по Леви пятимерные орбиты.

Доказательство. В первом случае леммы 3.2 рассмотрение тройки (6.2) для алгебры $\mathfrak{m}_{24}$ приводит к формуле

$$
e_{4}=\left(\left(A_{2}+1\right) z_{1}+A_{4}, B_{2} z_{1}+B_{4},-\frac{1}{2}\left(z_{1}-C_{2}\right)^{2}+2 w+C_{4}\right)
$$

с некоторыми комплексными константами $A_{4}, B_{4}, C_{4}$. Выписывая покомпонентно последний (из десяти) коммутатор $\left[e_{2}, e_{4}\right]=e_{2}$ обсуждаемой алгебры, получаем, с учетом формул (6.7) и (6.9), систему трех уравнений

$$
A_{2}\left(A_{2}+1\right)=A_{2}, \quad A_{2} B_{2}=B_{2}, \quad-A_{2}\left(z_{1}-C_{2}\right)-2\left(z_{1}-C_{2}\right)+\left(A_{2}+1\right) z_{1}+A_{4}=-\left(z_{1}-C_{2}\right) .
$$

Из этих равенств следует, что $A_{2}=B_{2}=0, A_{4}=-C_{2}$. В таком случае для поля $e_{2}$ получаем координатное представление

$$
e_{2}=\left(0,0,-\left(z_{1}-C_{2}\right)\right)
$$

Остается заметить, что наличие на обсуждаемой поверхности $M$ пары полей $e_{2}$ вида (6.10) и $e_{1}=(0,0,1)$ означает независимость определяющей функции $\Phi$ такой поверхности от переменной $w$. Как следствие, поверхность $M$ вырождена по Леви в первом случае.

Во втором случае леммы 3.2 мы имеем пять базисных полей вида

$$
\begin{aligned}
& e_{1}=(0,0,1), \\
& e_{3}=(1,0,0), \\
& e_{5}=\left(\hat{f}_{5}\left(z_{2}\right), 0, \hat{h}_{5}\left(z_{2}\right)\right), \\
& e_{2}=\left(f_{2}, g_{2}, h_{2}\right), \\
& e_{4}=\left(f_{4}, g_{4}, h_{4}\right) .
\end{aligned}
$$

Здесь из рассмотрения двух коммутаторов

$$
\left[e_{1}, e_{2}\right]=0, \quad\left[e_{2}, e_{3}\right]=e_{1}
$$


получаем упрощенный вид компонент поля

$$
e_{2}=\left(\hat{f}_{2}\left(z_{2}\right), \hat{g}_{2}\left(z_{2}\right),-z_{1}+\hat{h}_{2}\left(z_{2}\right)\right)
$$

и, в частности, факт их независимости от переменной $w$. Далее рассмотрим два подслучая, связанных с компонентой $\hat{g}_{2}\left(z_{2}\right)$ этого поля.

В первом подслучае, считая, что $\hat{g}_{2}\left(z_{2}\right) \equiv 0$ в некоторой окрестности обсуждаемой точки, мы получаем на поверхности $M$ четверку полей с нулевой второй компонентой

$$
\begin{aligned}
& e_{1}=(0,0,1), \\
& e_{3}=(1,0,0), \\
& e_{5}=\left(\hat{f}_{5}\left(z_{2}\right), 0, \hat{h}_{5}\left(z_{2}\right)\right), \\
& e_{2}=\left(\hat{f}_{2}, 0,-z_{1}+\hat{h}_{2}\right) .
\end{aligned}
$$

По лемме 3.4 такая поверхность $M$ вырождена по Леви.

Во втором подслучае будем считать, что функция $\hat{g}_{2}\left(z_{2}\right)$ принимает отличное от нуля значение (в исходной точке поверхности или вблизи нее). Тогда по замечанию 3.2 это поле можно упростить за счет голоморфной замены до состояния

$$
e_{2}=\left(0,1,-z_{1}\right)
$$

Поля $e_{1}, e_{3}, e_{5}$ при этом сохранят свой вид. Вычисляя с учетом формулы (6.11) коммутатор

$$
\left[e_{2}, e_{5}\right]=\left(\hat{f}_{5}^{\prime}, 0, \hat{h}_{5}^{\prime}\right)-\hat{f}_{5}(0,0,-1)=0
$$

получаем еще более упрощенный вид поля

$$
e_{5}=\left(A_{5}, 0,-A_{5} z_{2}+C_{5}\right),
$$

где $A_{5}, C_{5}$ - некоторые комплексные константы.

Рассмотрим в этой ситуации три коммутатора (6.2). Из равенства $\left[e_{1}, e_{4}\right]=2 e_{1}$ получаем первое упрощение поля $e_{4}$ до вида

$$
e_{4}=\left(f_{4}\left(z_{1}, z_{2}\right), g_{4}\left(z_{1}, z_{2}\right), 2 w+h_{4}\left(z_{1}, z_{2}\right)\right) .
$$

Второе из равенств (6.2) дает очередное его упрощение до состояния

$$
e_{4}=\left(z_{1}+\hat{f}_{4}\left(z_{2}\right), z_{1}+\hat{g}_{4}\left(z_{2}\right), 2 w-\frac{1}{2} z_{1}^{2}+\hat{h}_{4}\left(z_{2}\right)\right) \text {. }
$$

Переходя к последнему коммутатору из (6.2), получаем

$$
\left[e_{4}, e_{5}\right]=\left(z_{1}+\hat{g}_{4}\right)\left(0,0,-A_{5}\right)-\left(A_{5}\left(1,1,-z_{1}\right)+\left(-A_{5} z_{2}+C_{5}\right)(0,0,2)\right)=(0,0,0) .
$$

Из первой (как и из второй) компоненты этого равенства получается условие $A_{5}=0$. При таком условии касание полем (6.12) поверхности $M$ и наличие на этой поверхности поля $e_{1}=(0,0,1)$ возможно лишь (по аналогии с рассмотрениями первого случая) в ситуации вырожденной по Леви поверхности $M$. Предложение 6.1 доказано полностью.

6.2. Алгебра $\mathfrak{m}_{25}$. Коммутационные соотношения в алгебре $\mathfrak{m}_{25}$ имеют вид

$$
\left[e_{2}, e_{3}\right]=e_{1}, \quad\left[e_{1}, e_{4}\right]=(1+q) e_{1}, \quad\left[e_{2}, e_{4}\right]=e_{2}, \quad\left[e_{3}, e_{4}\right]=q e_{3}, \quad|q| \leqslant 1 .
$$

Предложение 6.2. Любая пятимерная Леви-невырожденная орбита голоморфной реализаиии любой алгебры из семейства $\mathfrak{m}_{25}$ либо является сферической поверхностью, либо голоморфно эквивалентна индефинитной трубке

$$
v=y_{1} y_{2}+y_{2} \ln y_{2}
$$

с афффинно-однородным основанием. 
Доказательство. Первый случай леммы 3.2. Рассмотрим тройку соотношений (6.3) для базиca (6.8)

$$
\begin{aligned}
& e_{1}=(0,0,1), \\
& e_{3}=(1,0,0), \\
& e_{5}=(0,1,0), \\
& e_{2}=\left(A_{2}, B_{2},-z_{1}+C_{2}\right), \\
& e_{4}=\left(f_{4}, g_{4}, h_{4}\right) .
\end{aligned}
$$

Из соотношения $\left[e_{4}, e_{5}\right]=0$ получаем независимость поля $e_{4}$ от переменной $z_{2}$. Соотношение $\left[e_{3}, e_{4}\right]=q e_{3}$ означает, что для компонент поля $e_{4}$ выполняются следующие равенства:

$$
\frac{\partial f_{4}}{\partial z_{1}}=q, \quad \frac{\partial g_{4}}{\partial z_{1}}=0, \quad \frac{\partial h_{4}}{\partial z_{1}}=0
$$

так что $e_{4}=\left(q z_{1}+\hat{f}_{4}(w), \hat{g}_{4}(w), \hat{h}_{4}(w)\right)$. Наконец, из $\left[e_{1}, e_{4}\right]=(1+q) e_{1}$ имеем:

$$
\frac{\partial \hat{f}_{4}}{\partial w}=0, \quad \frac{\partial \hat{g}_{4}}{\partial w}=0, \quad \frac{\partial \hat{h}_{4}}{\partial w}=(1+q) .
$$

Это означает, что предыдущая формула для $e_{4}$ еще более упростится и примет вид

$$
e_{4}=\left(q z_{1}+A_{4}, B_{4},(1+q) w+C_{4}\right) .
$$

На третьем шаге применения общей схемы в этом случае рассмотрим коммутатор $\left[e_{2}, e_{4}\right]=e_{2}$ для полей $e_{2}, e_{4}$, заданных, соответственно, формулами (6.7) и (6.13). Имеем здесь векторное равенство

$$
A_{2}(q, 0,0)+\left(-z_{1}+C_{2}\right)(0,0,1+q)-\left(q z_{1}+A_{4}\right)(0,0,-1)=\left(A_{2}, B_{2},-z_{1}+C_{2}\right) .
$$

Из отдельных компонент этого равенства получаем:

$$
A_{2} q=A_{2}, \quad 0=B_{2}, \quad\left(-z_{1}+C_{2}\right)(1+q)+\left(q z_{1}+A_{4}\right)=-z_{1}+C_{2} .
$$

Это означает, что

$$
A_{2}(q-1)=0, \quad B_{2}=0, \quad A_{4}=-q C_{2} .
$$

Первое из этих равенств порождает два типа базисов в случае 1 , а именно базисы

$$
\begin{array}{ll}
e_{1}=(0,0,1), & e_{1}=(0,0,1), \\
e_{3}=(1,0,0), & e_{3}=(1,0,0), \\
e_{5}=(0,1,0), & \text { и } \quad e_{5}=(0,1,0), \\
e_{2}=\left(0,0,-z_{1}+C_{2}\right), & e_{2}=\left(A_{2}, 0,-z_{1}+C_{2}\right), \\
e_{4}=\left(q\left(z_{1}-C_{2}\right), B_{4},(1+q) w+C_{4}\right) & e_{4}=\left(z_{1}-C_{2}, B_{4}, 2 w+C_{4}\right) .
\end{array}
$$

Отметим, впрочем, что первый тип из-за наличия в базисе векторов $e_{1}=(0,0,1), e_{2}=\left(0,0,-z_{1}+\right.$ $C_{2}$ ) приводит к независимости определяющей функции изучаемых поверхностей от переменной $w$, а значит, к их вырожденности по Леви. Поэтому имеет смысл рассмотрение лишь второго типа, удовлетворяющего условию $q=1$. Располагая поля базиса естественным образом и производя сдвиги переменных

$$
z_{1}^{*}=z_{1}-C_{2}, \quad w^{*}=w-\frac{C_{4}}{2},
$$

получим (после отбрасывания знаков «*») базис

$$
\begin{aligned}
& e_{1}=(0,0,1), \\
& e_{2}=\left(A_{2}, 0,-z_{1}\right), \\
& e_{3}=(1,0,0), \\
& e_{4}=\left(z_{1}, B_{4}, 2 w\right), \\
& e_{5}=(0,1,0) .
\end{aligned}
$$


Замечание 6.1. Можно считать, что здесь параметры $A_{2}$ и $B_{4}$ чисто мнимые (за счет рассмотрения вместо полей $e_{2}, e_{4}$ их линейных комбинаций, соответственно, с полями $e_{3}$ и $\left.e_{5}\right)$. Введем для них упрощенные обозначения: $A_{2}=i a, B_{4}=i b(a, b \in \mathbb{R})$.

Ясно, что любая интегральная поверхность алгебры с базисом (6.14) является трубчатой поверхностью, уравнение которой $\Phi\left(y_{1}, y_{2}, v\right)=0$ не зависит от вещественных координат $x_{1}, x_{2}, u$ и может быть записано в виде

$$
v=F\left(y_{1}, y_{2}\right) .
$$

Содержательная часть системы (двух оставшихся) уравнений в частных производных, отвечающая такой алгебре, имеет, как несложно проверить, следующий вид

$$
a \frac{\partial F}{\partial y_{1}}+y_{1}=0, \quad y_{1} \frac{\partial F}{\partial y_{1}}+b \frac{\partial F}{\partial y_{2}}-2 F=0 .
$$

Ее интегрирование не представляет большого труда. Случай $a=0$, в котором поля $e_{1}$ и $e_{2}$ пропорциональны, мы не рассматриваем. При $a \neq 0$ из первого уравнения получаем

$$
F=-\frac{1}{2 a} y_{1}^{2}+G\left(y_{2}\right)
$$

с произвольной аналитической функцией $G\left(y_{2}\right)$. Подстановка этой формулы во второе уравнение системы дает нам ОДУ

$$
b G^{\prime}\left(y_{2}\right)=2 G\left(y_{2}\right),
$$

имеющее общим решением функцию $G\left(y_{2}\right)=C e^{(2 / b) y_{2}}$ с произвольной константой $C$. Тогда с точностью до очевидных растяжений комплексных переменных все невырожденные по Леви однородные поверхности, отвечающие алгебрам с базисами случая 1 , совпадают с трубками

$$
v=y_{1}^{2} \pm e^{y_{2}} .
$$

Обе эти поверхности, как известно (см., например, $[6,19])$, являются сферическими, т.е. каждая из них голоморфно эквивалентна одной из (аффинно-однородных) квадрик (1.1).

Второй случай леммы 3.2. В данном случае рассмотрения можно начинать с базиса

$$
\begin{aligned}
& e_{1}=(0,0,1), \\
& e_{3}=(1,0,0), \\
& e_{5}=\left(A_{5}, 0,-A_{5} z_{2}+C_{5}\right), \\
& e_{2}=\left(0,1,-z_{1}\right), \\
& e_{4}=\left(f_{4}, g_{4}, h_{4}\right),
\end{aligned}
$$

полученного при рассмотрении алгебры $\mathfrak{m}_{24}$ (за счет использования трех «универсальных» коммутационных соотношений).

Далее рассмотрим три коммутатора $\left[e_{1}, e_{4}\right],\left[e_{3}, e_{4}\right],\left[e_{4}, e_{5}\right]$. Из соотношения $\left[e_{1}, e_{4}\right]=(1+q) e_{1}$ имеем формулу

$$
e_{4}=\left(f_{4}(z), g_{4}(z),(1+q) w+h_{4}(z)\right) .
$$

Равенство $\left[e_{3}, e_{4}\right]=q e_{3}$ позволяет уточнить формулу (6.16) до вида

$$
e_{4}=\left(q z_{1}+\hat{f}_{4}\left(z_{2}\right), \hat{g}_{4}\left(z_{2}\right),(1+q) w+\hat{h}_{4}\left(z_{2}\right)\right) .
$$

Тогда коммутатор $\left[e_{4}, e_{5}\right]=0$ дает нам векторное равенство

$$
\hat{g}_{4}\left(0,0,-A_{5}\right)-A_{5}(q, 0,0)-\left(-A_{5} z_{2}+C_{5}\right)(0,0,1+q)=(0,0,0) .
$$

Вторая компонента этого равенства выполняется тождественно, а первая и третья имеют вид

$$
A_{5} q=0, \quad-g_{4} A_{5}+(1+q)\left(A_{5} z_{2}-C_{5}\right)=0 .
$$

Заметим, что при $A_{5}=0$ наличие пары полей $e_{1}=(1,0,0)$ и $e_{5}=\left(0,0, C_{5}\right)$ гарантированно сводит обсуждение либо к неполному рангу алгебры, либо к вырождению по Леви интегральной поверхности. Интересуясь только невырожденным случаем, можно считать поэтому, что

$$
q=0, \quad e_{4}=\left(\hat{f}_{4}\left(z_{2}\right), z_{2}-\frac{C_{5}}{A_{5}}, w+\hat{h}_{4}\left(z_{2}\right)\right) .
$$


Последний коммутатор $\left[e_{2}, e_{4}\right]=e_{2}$ дает теперь очередное векторное ограничение

$$
\left(\hat{f}_{4}^{\prime}, 1, \hat{h}_{4}^{\prime}\right)-z_{1}(0,0,1)-\hat{f}_{4}(0,0,-1)=\left(0,1,-z_{1}\right) .
$$

Из него следует, что

$$
\hat{f}_{4}=A_{4}=\text { const }, \quad \hat{h}_{4}=-A_{4} z_{2}+C_{4}
$$

с некоторыми комплексными константами $A_{4}, C_{4}$, а еще одно тождественное скалярное равенство $1=1$ составляет третью компоненту (6.17).

В итоге после сдвигов $z_{2}^{*}=z_{2}-C_{5} / A_{5}, w^{*}=w+C_{4}^{*}$ мы получаем (отбрасывая знаки «*») следующий вид базиса алгебры $\mathfrak{m}_{25}$ во втором случае:

$$
\begin{aligned}
& e_{1}=(0,0,1), \\
& e_{3}=(1,0,0), \\
& e_{5}=\left(A_{5}, 0,-A_{5} z_{2}\right), \\
& e_{2}=\left(0,1,-z_{1}\right), \\
& e_{4}=\left(A_{4}, z_{2}, w-A_{4} z_{2}\right) .
\end{aligned}
$$

Любая такая алгебра оказывается аффинной и, следовательно, все интегральные поверхности таких алгебр являются афбфинно-однородными. Этого достаточно для подтверждения справедливости теоремы 1.1 в рассматриваемом случае 2 семейства алгебр $\mathfrak{m}_{25}$.

Прокомментируем кратко решение системы трех уравнений в частных производных, соответствующей алгебре Ли с таким базисом и уравнениям искомых однородных поверхностей вида $v=F\left(y_{1}, x_{2}, y_{2}\right)$. После разложения коэффициентов $A_{4}, A_{5}$ на вещественные и мнимые части $A_{k}=a_{k}+i b_{k}\left(a_{k}, b_{k} \in \mathbb{R}, k=4,5\right)$ эта система принимает вид

$$
b_{5} \frac{\partial F}{\partial y_{1}}+\left(b_{5} x_{2}+a_{5} y_{2}\right)=0, \quad \frac{\partial F}{\partial x_{2}}=-y_{1}, \quad b_{4} \frac{\partial F}{\partial y_{1}}+x_{2} \frac{\partial F}{\partial x_{2}}+y_{2} \frac{\partial F}{\partial y_{2}}=F-\left(b_{4} x_{2}+a_{4} y_{2}\right) .
$$

Подробную процедуру решения этой системы мы здесь не приводим. Отметим лишь, что наиболее значимым среди ее коэффициентов является $b_{5}$ : при нулевом значении этого коэффициента однородные поверхности, связанные с базисом (6.18) и системой (6.19), являются вырожденными по Леви. При ненулевом $b_{5}$ в процессе решения системы возникает параметр $R=a_{5} / b_{5}$, входящий в итоговую формулу для уравнений искомых однородных поверхностей. После простейших аффинных замен переменных эта формула приобретет вид

$$
v=y_{1}\left(x_{2}-R y_{2}\right)+y_{2} \ln y_{2} .
$$

Однако квадратичная замена переменной $w^{*}=w-z_{1} z_{2}$ преобразует уравнение $(6.20)$ в

$$
v+x_{1} y_{2}+x_{2} y_{1}=y_{1}\left(x_{2}-R y_{2}\right)+y_{2} \ln y_{2} \Longleftrightarrow v=-y_{2}\left(x_{1}+R y_{1}\right)+y_{2} \ln y_{2} .
$$

Еще одно аффинное преобразование $z_{1}^{*}=-(i+R) z_{1}$ превращает его в уравнение

$$
v=y_{1} y_{2}+y_{2} \ln y_{2}
$$

индефинитной аффинно-однородной трубки, имеющей семимерную алгебру симметрий. На этом доказательство предложения 6.2 завершено.

6.3. Алгебра $\mathfrak{m}_{26}$. Коммутационные соотношения в алгебре $\mathfrak{m}_{26}$ имеют вид

$$
\left[e_{1}, e_{4}\right]=2 p e_{1}, \quad\left[e_{2}, e_{3}\right]=e_{1}, \quad\left[e_{2}, e_{4}\right]=p e_{2}-e_{3}, \quad\left[e_{3}, e_{4}\right]=e_{2}+p e_{3} \quad(p \geqslant 0) .
$$

Предложение 6.3. Любая пятимерная Леви-невырожденная орбита голоморфной реализа-

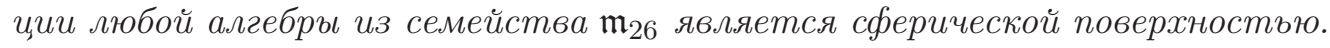

Доказательство. Первый случай леммы 3.2. Здесь удобно считать, что все три поля $e_{1}, e_{3}, e_{5}$ выпрямлены до состояния

$$
e_{1}=(0,0,1), \quad e_{3}=(0,1,0), \quad e_{5}=(1,0,0) .
$$

Такой их вид означает, в частности, что все обсуждаемые в этом случае интегральные поверхности алгебры $\mathfrak{m}_{26}$ являются трубчатыми поверхностями. 
Для упрощения вида двух оставшихся полей (и, тем самым, для получения формул, описывающих основания обсуждаемых трубок) воспользуемся коммутационными соотношениями, имеющимися в алгебре $\mathfrak{m}_{26}$. Так, из поочередного рассмотрения коммутаторов

$$
\left[e_{1}, e_{2}\right]=0, \quad\left[e_{2}, e_{5}\right]=0, \quad\left[e_{2}, e_{3}\right]=e_{1}
$$

получаем для поля $e_{2}$, имевшего первоначально общий вид

$$
e_{2}=\left(f_{2}\left(z_{1}, z_{2}, w\right), g_{2}\left(z_{1}, z_{2}, w\right), h_{2}\left(z_{1}, z_{2}, w\right)\right),
$$

постепенные упрощения. Из равенства

$$
\left(\frac{\partial f_{2}}{\partial w}, \frac{\partial g_{2}}{\partial w}, \frac{\partial h_{2}}{\partial w}\right)=(0,0,0)
$$

имеем

$$
e_{2}=\left(f_{2}\left(z_{1}, z_{2}\right), g_{2}\left(z_{1}, z_{2}\right), h_{2}\left(z_{1}, z_{2}\right)\right) .
$$

Аналогично, за счет рассмотрения коммутатора $\left[e_{2}, e_{5}\right]=0$, компоненты поля $e_{2}$ освобождаются от переменной $z_{1}$, так что

$$
e_{2}=\left(\hat{f}_{2}\left(z_{2}\right), \hat{g}_{2}\left(z_{2}\right), \hat{h}_{2}\left(z_{2}\right)\right) \text {. }
$$

Тогда из $\left[e_{2}, e_{3}\right]=e_{1}$ получаем

$$
-\left(\hat{f}_{2}^{\prime}\left(z_{2}\right), \hat{g}_{2}^{\prime}\left(z_{2}\right), \hat{h}_{2}^{\prime}\left(z_{2}\right)\right)=(0,0,1),
$$

откуда

$$
e_{2}=\left(A_{2}, B_{2},-z_{2}+C_{2}\right)
$$

с некоторыми комплексными константами $A_{2}, B_{2}, C_{2}$. Аналогичное поочередное рассмотрение тройки коммутаторов

$$
\left[e_{1}, e_{4}\right]=2 p e_{1}, \quad\left[e_{4}, e_{5}\right]=0, \quad\left[e_{3}, e_{4}\right]=e_{2}+p e_{3}
$$

упрощает поле $e_{4}$ до вида

$$
e_{4}=\left(A_{2} z_{2}+A_{4},\left(p+B_{2}\right) z_{2}+B_{4}, 2 p w-\frac{1}{2} z_{2}^{2}+C_{2} z_{2}+C_{4}\right) .
$$

В самом деле, сначала получаем

$$
e_{4}=\left(f_{4}\left(z_{1}, z_{2}\right), g_{4}\left(z_{1}, z_{2}\right), 2 p w+h_{4}\left(z_{1}, z_{2}\right)\right) .
$$

Затем из соотношения $\left[e_{4}, e_{5}\right]=0$ имеем

$$
e_{4}=\left(\hat{f}_{4}\left(z_{2}\right), \hat{g}_{4}\left(z_{2}\right), 2 p w+\hat{h}_{4}\left(z_{2}\right)\right) .
$$

Наконец, используя такой вид поля $e_{4}$ при рассмотрении коммутатора $\left[e_{3}, e_{4}\right]=e_{2}+p e_{3}$, приходим к векторному равенству

$$
\left(\hat{f}_{4}^{\prime}, \hat{g}_{4}^{\prime}, \hat{h}_{4}^{\prime}\right)=\left(A_{2}, B_{2}+p,-z_{2}+C_{2}\right) .
$$

Интегрируя его покомпонентно, получаем (6.22).

Остается рассмотреть последнее коммутационное соотношение $\left[e_{2}, e_{4}\right]=p e_{2}-e_{3}$ для полученных полей $e_{2}$ и $e_{4}$, т.е.

$$
\begin{array}{r}
B_{2}\left(A_{2}, p+B_{2},-z_{2}+C_{2}\right)+\left(-z_{2}+C_{2}\right)(0,0,2 p)-\left(\left(p+B_{2}\right) z_{2}+B_{4}\right)\left(0,0,-z_{2}+C_{2}\right)= \\
=\left(p A_{2}, p B_{2}-1, p\left(-z_{2}+C_{2}\right)\right) .
\end{array}
$$

В трех отдельных компонентах этого векторного равенства получаем

$$
\begin{gathered}
B_{2} A_{2}=p A_{2}, \quad B_{2}\left(p+B_{2}\right)=p B_{2}-1, \\
B_{2}\left(-z_{2}+C_{2}\right)+2 p\left(-z_{2}+C_{2}\right)+\left(\left(p+B_{2}\right) z_{2}+B_{4}\right)=p\left(-z_{2}+C_{2}\right)
\end{gathered}
$$

или

$$
B_{2}^{2}=-1, \quad A_{2}\left(B_{2}-p\right)=0, \quad B_{4}=-C_{2}\left(B_{2}+p\right) .
$$


Таким образом, в первом случае базис голоморфной реализации алгебры $\mathfrak{m}_{26}$ можно представить в виде

$$
\begin{aligned}
& e_{1}=(0,0,1), \\
& e_{2}=\left(0, i \varepsilon,-z_{2}+C_{2}\right), \\
& e_{3}=(0,1,0), \\
& e_{4}=\left(A_{4},(p+i \varepsilon)\left(z_{2}-C_{2}\right), 2 p w-\left(z_{2}-C_{2}\right)^{2} / 2+C_{4}^{*}\right), \\
& e_{5}=(1,0,0),
\end{aligned}
$$

где $C_{4}^{*}=C_{4}+C_{2}^{2} / 2, \varepsilon= \pm 1$.

Осуществим сдвиг переменной $z_{2}-C_{2}=z_{2}^{*}$ и отбросим знак «*» у этой переменной и у коэффициента $C_{4}^{*}$. С учетом чисто мнимого характера коэффициентов $A_{4}=i a_{4}, C_{4}=i c_{4}$, получаем существенно упрощенный вид такого базиса:

$$
\begin{aligned}
& e_{1}=(0,0,1), \\
& e_{2}=\left(0, i \varepsilon,-z_{2}\right), \\
& e_{3}=(0,1,0), \\
& e_{4}=\left(i a_{4},(p+i \varepsilon) z_{2}, 2 p w-z_{2}^{2} / 2+i c_{4}\right), \\
& e_{5}=(1,0,0) .
\end{aligned}
$$

Система уравнений в частных производных, отвечающая двум нетривиальным полям из набора (6.24) и уравнению $v=F\left(y_{1}, y_{2}\right)$ любой искомой трубчатой поверхности, имеет вид

$$
\varepsilon \frac{\partial F}{\partial y_{2}}=-y_{2}, \quad a_{4} \frac{\partial F}{\partial y_{1}}+\left(\varepsilon x_{2}+p y_{2}\right) \frac{\partial F}{\partial y_{2}}=2 p F-x_{2} y_{2}+c_{4} .
$$

Заметим, что группа слагаемых из второго уравнения, содержащих множителем $x_{2}$, удаляется из него за счет использования первого уравнения. Еще одно соображение касается обращения в нуль хотя бы одного из двух коэффициентов $a_{4}$ или $p$ этого уравнения. В каждом из этих случаев интегральная поверхность обсуждаемой алгебры Ли, отвечающая решениям системы (6.25), оказывается, как несложно проверить, вырожденной по Леви. При ненулевых $a_{4}$ и $p$ решение системы (6.25) также не представляет сложностей. Сначала получаем

$$
F=-\frac{\varepsilon}{2} y_{2}^{2}+G\left(y_{1}\right)
$$

Функция $G\left(y_{1}\right)$ с точностью до постоянного слагаемого равна $\exp \left(2 p y_{1} / a_{4}\right)$. Это означает, что уравнения невырожденных однородных поверхностей, отвечающих первому случаю для алгебры $\mathfrak{m}_{26}$, имеют (с точностью до тривиальных аффинных преобразований) вид

$$
v=y_{2}^{2} \pm e^{y_{1}} .
$$

Как было отмечено выше (см. формулу (6.15)), такие поверхности являются сферическими.

Замечание 6.2. Совпадение (с точностью до голоморфной эквивалентности) интегральных поверхностей, отвечающих разным пятимерным алгебрам $\mathfrak{m}_{25}$ и $\mathfrak{m}_{26}$, вполне объяснимо. Обе эти алгебры являются подалгебрами 15-мерных алгебр квадрик (1.1).

Второй случай леммы 3.2. Здесь мы воспользуемся тем, что у любой алгебры со структурой $\mathfrak{m}_{26}$ можно указать две различные абелевы подалгебры. Помимо обсуждавшейся выше линейной оболочки $\left\langle e_{1}, e_{3}, e_{5}\right\rangle$ абелевой подалгеброй является и $\left\langle e_{1}, e_{2}, e_{5}\right\rangle$. Поэтому, имея упрощенный до вида

$$
\begin{aligned}
& e_{1}=(0,0,1), \\
& e_{5}=(1,0,0), \\
& e_{3}=\left(\hat{f}_{3}, 0, \hat{h}_{3}\right)
\end{aligned}
$$

базис одной из этих двух подалгебр, обсудим дополнительное упрощение поля $e_{2}$. Из двух соотношений

$$
\left[e_{1}, e_{2}\right]=0, \quad\left[e_{2}, e_{5}\right]=0
$$


легко получается следующее представление:

$$
e_{2}=\left(\hat{f}_{2}, \hat{g}_{2}, \hat{h}_{2}\right)
$$

Если при этом функция $\hat{g}_{2}\left(z_{2}\right)$ тождественно равна нулю (в некоторой окрестности начала координат), то все интегральные поверхности алгебры с таким базисом оказываются вырожденными.

Рассматривая случай алгебр с невырожденными интегральными поверхностями, будем считать что $\hat{g}_{2}(0) \neq 0$. Тогда, пользуясь техникой, предложенной в [16], выпрямим поле $e_{2}$ до состояния

$$
e_{2}=(0,1,0)=\frac{\partial}{\partial z_{2}}
$$

Выпрямленные поля $e_{1}, e_{5}$, а также поле $e_{3}$ сохранят при этом свой упрощенный вид.

Дальнейшие упрощения базиса проводим по стандартной схеме. Из равенства $\left[e_{2}, e_{3}\right]=e_{1}$ с учетом (6.26) получим представление поля

$$
e_{3}=\left(A_{3}, 0, z_{2}+C_{3}\right) .
$$

Рассматривая теперь коммутаторы $\left[e_{1}, e_{4}\right]=2 p e_{1},\left[e_{4}, e_{5}\right]=0$ с участием поля $e_{4}$, имеем

$$
e_{4}=\left(\hat{f}_{4}, \hat{g}_{4}, 2 p w+\hat{h}_{4}\right) \text {. }
$$

Тогда равенство $\left[e_{2}, e_{4}\right]=p e_{2}-e_{3}$ означает, что

$$
\left(\hat{f}_{4}^{\prime}, \hat{g}_{4}^{\prime}, \hat{h}_{4}^{\prime}\right)=\left(-A_{3}, p,-\left(z_{2}+C_{3}\right)\right),
$$

так что

$$
e_{4}=\left(-A_{3} z_{2}+A_{4}, p z_{2}+B_{4}, 2 p w-\frac{1}{2}\left(z_{2}+C_{3}\right)^{2}+C_{4}\right) .
$$

Остается теперь заметить, что последнее из имеющихся в обсуждаемой алгебре коммутационных соотношений

$$
\left[e_{3}, e_{4}\right]=e_{2}+p e_{3}
$$

является в такой ситуации противоречивым, так как вторая компонента векторного равенства (6.27) имеет вид $0=1$. Это противоречие означает, что во втором случае алгебры со структурой $\mathfrak{m}_{26}$ не имеют реализаций с невырожденными по Леви однородными гиперповерхностями. Предложение 6.3 доказано.

7. Голоморфные реализации алгебры $\mathfrak{m}_{27}$. Алгебра $\mathfrak{m}_{27}$ с четырьмя нетривиальными коммутационными соотношениями

$$
\left[e_{1}, e_{3}\right]=e_{1}, \quad\left[e_{2}, e_{3}\right]=e_{2}, \quad\left[e_{1}, e_{4}\right]=-e_{2}, \quad\left[e_{2}, e_{4}\right]=e_{1}
$$

имеет абелев идеал $\left\langle e_{1}, e_{2}, e_{5}\right\rangle$. Выпрямление полей и рассмотрение связанных с ним двух случаев мы начнем именно с базисной тройки этого идеала.

Предложение 7.1. Любая пятимерная Леви-невырожденная орбита голоморфной реализаиии алгебры 'ำ либо является индефинитной сберической поверхностъю, либо голоморфно эквивалентна одной из поверхностей семейства

$$
v=\alpha \arg \left(y_{1}+i y_{2}\right)+\ln \left(y_{1}^{2}+y_{2}^{2}\right), \quad \alpha \in \mathbb{R} .
$$

Замечание 7.1. Индефинитные аффинно-однородные поверхности (7.1) имеются в известном списке [20]. Отметим, кроме того, что размерность максимальной алгебры голоморфных векторных полей, связанной с каждой из этих поверхностей, равна 7 (см. [21]).

Доказательство предложения 7.1.

Первый случай леммы 3.2. Считаем, что все три поля $e_{1}, e_{2}, e_{5}$ выпрямлены до состояния

$$
e_{1}=(0,0,1), \quad e_{2}=(0,1,0), \quad e_{5}=(1,0,0) .
$$

Из поочередного рассмотрения коммутаторов

$$
\left[e_{1}, e_{3}\right]=e_{1}, \quad\left[e_{3}, e_{5}\right]=0, \quad\left[e_{2}, e_{3}\right]=e_{2}
$$


получаем постепенные упрощения поля $e_{3}$ :

$$
\begin{array}{r}
e_{3}=\left(f_{3}\left(z_{1}, z_{2}\right), g_{3}\left(z_{1}, z_{2}\right), w+h_{3}\left(z_{1}, z_{2}\right),\right. \\
e_{3}=\left(\hat{f}_{3}\left(z_{2}\right), \hat{g}_{3}\left(z_{2}\right), w+\hat{h}_{3}\left(z_{2}\right)\right), \\
e_{3}=\left(A_{3}, z_{2}+B_{3}, w+C_{3}\right)
\end{array}
$$

с некоторыми комплексными константами $A_{3}, B_{3}, C_{3}$. Аналогичное рассмотрение тройки коммутаторов

$$
\left[e_{1}, e_{4}\right]=-e_{2}, \quad\left[e_{4}, e_{5}\right]=0, \quad\left[e_{2}, e_{4}\right]=e_{1}
$$

приводит к формуле

$$
e_{4}=\left(A_{4},-w+B_{4}, z_{2}+C_{4}\right) .
$$

Из последнего имеющегося в алгебре $\mathfrak{m}_{27}$ соотношения $\left[e_{3}, e_{4}\right]=0$ выводятся равенства

$$
C_{3}+B_{4}=0, \quad B_{3}-C_{4}=0
$$

и очередное упрощение пары полей $e_{3}$ и $e_{4}$ :

$$
e_{3}=\left(A_{3}, z_{2}+B_{3}, w+C_{3}\right), \quad e_{4}=\left(A_{4},-\left(w+C_{3}\right), z_{2}+B_{3}\right) .
$$

После сдвига переменных $z_{2}^{*}=z_{2}+B_{3}, w^{*}=w+C_{3}$ набор базисных полей алгебры $\mathfrak{m}_{27}$ примет вид

$$
\begin{aligned}
& e_{1}=(0,0,1), \\
& e_{2}=(0,1,0), \\
& e_{5}=(1,0,0), \\
& e_{3}=\left(A_{3}, z_{2}, w\right), \\
& e_{4}=\left(A_{4},-w, z_{2}\right) .
\end{aligned}
$$

Рассматривая при этом вместо полей $e_{3}, e_{4}$ их комбинации с полем $e_{5}$, можно считать константы $A_{3}, A_{4}$ в (7.3) чисто мнимыми. Обозначим для удобства $A_{3}=i a_{3}, A_{4}=i a_{4}\left(a_{3}, a_{4} \in \mathbb{R}\right)$.

Любая орбита алгебры с базисом (7.3), очевидно, является трубчатой. Основание такой трубки является аффинно однородным (в силу коммутирования полей $e_{3}$ и $e_{4}$ ) и удовлетворяет следующей достаточно простой системе двух уравнений в частных производных относительно определяющей функции $v=F\left(y_{1}, y_{2}\right)$ :

$$
a_{3} \frac{\partial F}{\partial y_{1}}+y_{2} \frac{\partial F}{\partial y_{2}}=F, \quad a_{4} \frac{\partial F}{\partial y_{1}}-F \frac{\partial F}{\partial y_{2}}=y_{2} .
$$

Отметим, что при одновременном обращении в нуль обоих коэффициентов $a_{3}, a_{4}$ любая интегральная поверхность алгебры (7.3) является вырожденной в силу леммы 3.4. Рассмотрение ненулевых наборов $\left(a_{3}, a_{4}\right)$ естественно разделить (за счет возможного растяжения переменной $\left.z_{1}\right)$ на два подслучая: (i) $a_{3}=0, a_{4}=1$ и (ii) $a_{3}=1, a_{4} \in \mathbb{R}$.

В первом из них стандартным методом последовательного решения отдельных уравнений системы (7.4) приходим к уравнению сферической (см. [6]) однородной поверхности

$$
v=y_{2} \operatorname{tg} y_{1} .
$$

Во втором подслучае аналогично получаем более сложное уравнение (7.1).

Замечание 7.2. Уравнение (7.5) легко преобразуется в $y_{1}=\operatorname{arctg}\left(v / y_{2}\right)$ или, с точностью до переобозначения переменных, в полученное в (5.12) уравнение поверхности $v=\arg \left(y_{1}+i y_{2}\right)$.

Замечание 7.3. С точностью до голоморфных преобразований полученные в первом случае невырожденные орбиты алгебры $\mathfrak{m}_{27}$ можно объединить в семейство

$$
v=\alpha \arg \left(y_{1}+i y_{2}\right)+\beta \ln \left(y_{1}^{2}+y_{2}^{2}\right),
$$

элементы которого описываются одномерным проективным параметром $(\alpha: \beta)$. 
Второй случай леммы 3.2. Тройку базисных полей абелева идеала алгебры $\mathfrak{m}_{27}$ представим в виде

$$
e_{1}=(0,0,1), \quad e_{2}=(1,0,0), \quad e_{5}=\left(\hat{f}_{5}, 0, \hat{h}_{5}\right) .
$$

Для (частичного) упрощения поля $e_{3}$ обсуждаемой алгебры рассмотрим два коммутационных соотношения

Из них следует, что

$$
\left[e_{1}, e_{3}\right]=e_{1}, \quad\left[e_{2}, e_{3}\right]=e_{2} .
$$

$$
e_{3}=\left(z_{1}+\hat{f}_{3}\left(z_{2}\right), \hat{g}_{3}\left(z_{2}\right), w+\hat{h}_{3}\left(z_{2}\right)\right) .
$$

Отметим при этом, что тождественное обращение в нуль функции $\hat{g}_{3}\left(z_{2}\right)$ означало бы наличие в обсуждаемой алгебре четверки базисных (а потому линейно независимых) полей с тождественно нулевыми вторыми компонентами. Это приводит (согласно лемме 3.4) к вырождению по Леви любой интегральной поверхности обсуждаемой алгебры Ли. Поэтому далее можно считать (возможно, после сдвига начала координат), что $\hat{g}_{3}(0) \neq 0$. В такой ситуации мы упростим поле $e_{3}$. Пользуясь техникой [16] (см. замечание 3.2 ), голоморфной заменой его можно привести к виду $e_{3}=\left(z_{1}, 1, w\right)$. При этом вид выпрямленных полей $e_{1}, e_{2}$, а также упрощенный вид поля $e_{5}$ сохранятся. Более того, из коммутационного соотношения $\left[e_{3}, e_{5}\right]=0$ теперь следует, что

$$
\left(\hat{f}_{5}^{\prime}, 0, \hat{h}_{5}^{\prime}\right)-\hat{f}_{5}(1,0,0)-\hat{h}_{5}(0,0,1)=(0,0,0)
$$

или

$$
e_{5}=\left(A_{5} e^{z_{2}}, 0, C_{5} e^{z_{2}}\right)
$$

с некоторыми комплексными коэффициентами $A_{5}, C_{5}$.

Далее обсудим четыре оставшихся коммутатора в алгебре $\mathfrak{m}_{27}$. Все они содержат в качестве множителя $e_{4}$ и имеют вид

$$
\left[e_{1}, e_{4}\right]=-e_{2}, \quad\left[e_{2}, e_{4}\right]=e_{1}, \quad\left[e_{3}, e_{4}\right]=0, \quad\left[e_{4}, e_{5}\right]=0 .
$$

Из первой тройки этих соотношений следует, что

$$
e_{4}=\left(-w+A_{4} e^{z_{2}}, B_{4}, z_{1}+C_{4} e^{z_{2}}\right)
$$

с некоторыми комплексными коэффициентами $A_{4}, B_{4}, C_{4}$. Тогда последнее соотношение из (7.6) означает, что

$$
B_{4}\left(A_{5} e^{z_{2}}, 0, C_{5} e^{z_{2}}\right)-A_{5} e^{z_{2}}(0,0,1)-C_{5} e^{z_{2}}(-1,0,0)=(0,0,0) .
$$

Из двух содержательных компонент этого векторного равенства получаем итоговые ограничения на коэффициенты пары полей $e_{4}, e_{5}$ :

$$
B_{4} A_{5}+C_{5}=0, \quad B_{4} C_{5}-A_{5}=0 \quad \text { или } \quad B_{4}^{2}=-1, C_{5}=-B_{4} A_{5} .
$$

В итоге набор пяти базисных полей алгебры $\mathfrak{m}_{27}$ имеет во втором случае вид

$$
\begin{aligned}
& e_{1}=(0,0,1), \\
& e_{2}=(1,0,0), \\
& e_{3}=\left(z_{1}, 1, w\right), \\
& e_{4}=\left(-w+A_{4} e^{z_{2}}, i \varepsilon, z_{1}+C_{4} e^{z_{2}}\right), \\
& e_{5}=\left(A_{5} e^{z_{2}}, 0,-i \varepsilon A_{5} e^{z_{2}}\right)
\end{aligned}
$$

с некоторыми комплексными коэффициентами $A_{4}, C_{4}, A_{5}\left(A_{5} \neq 0\right), \varepsilon= \pm 1$.

Этот вид можно еще упростить несложными голоморфными преобразованиями. Во-первых, представим ненулевой коэффициент $A_{5}$ в тригонометрической форме $A_{5}=r e^{i t}, t \in[0,2 \pi)$. Рассматривая тогда вместо поля $e_{5}$ пропорциональное ему поле $(1 / r) e_{5}$, мы сможем записать его в виде

$$
e_{5}=\left(e^{z_{2}+i t}, 0,-i \varepsilon e^{z_{2}+i t}\right) .
$$

Сдвиг переменной $z_{2}^{*}=z_{2}+i t$ переводит это поле в $e_{5}=\left(e^{z_{2}}, 0,-i \varepsilon e^{z_{2}}\right)$ и сохраняет вид остальных полей базиса (7.7) (с измененными значениями коэффициентов $A_{4}, C_{4}$ ). 
Еще одной, экспоненциальной, заменой $z_{2}^{*}=e^{z_{2}}$ алгебра с базисом (7.7) превращается в алгебру аффинных векторных полей с базисом

$$
\begin{aligned}
& e_{1}=(0,0,1), \\
& e_{2}=(1,0,0), \\
& e_{3}=\left(z_{1}, z_{2}, w\right), \\
& e_{4}=\left(-w+A_{4} z_{2}, i \varepsilon z_{2}, z_{1}+C_{4} z_{2}\right), \\
& e_{5}=\left(z_{2}, 0,-i \varepsilon z_{2}\right) .
\end{aligned}
$$

Кроме того, рассматривая вместо поля $e_{4}$ комбинацию $e_{4}-\left(\operatorname{Im} A_{4}\right) e_{5}$, коэффициент $A_{4}$ в формулах (7.8) можно считать чисто мнимым. Тем самым, любая интегральная поверхность алгебры $\mathfrak{m}_{27}$, реализованной по схеме второго случая, является (с точностью до голоморфных преобразований) аффинно однородной поверхностью. Этим утверждением завершается доказательство предложения 7.1 и основной теоремы 1.1.

Приведем здесь описание процедуры интегрирования алгебр (7.8) и вывод о получаемых при этом однородных поверхностях.

Предложение 7.2. При произвольных значениях параметров $A_{4}, C_{4}$ любая интегральная поверхность алгебры (7.8) голоморфно эквивалентна индефинитной квадрике

$$
v=\left|z_{1}\right|^{2}-\left|z_{2}\right|^{2}
$$

Доказательство. Выделим, прежде всего, вещественные и мнимые части коэффициентов набора полей (7.8). С учетом сказанного, можно представить эти коэффициенты в виде

$$
A_{4}=i b_{4}, \quad C_{4}=c_{4}+i d_{4},
$$

где $b_{4}, c_{4}, d_{4} \in \mathbb{R}$. Как и ранее, определяющую функцию любой искомой поверхности (интегральной для произвольной алгебры с базисом вида (7.8)) считаем имеющей вид $v=F\left(y_{1}, x_{2}, y_{2}\right)$. Содержательная система уравнений в частных производных, связанная с нахождением этой функции, определяется тремя полями $e_{3}, e_{4}, e_{5}$ :

$$
\begin{array}{ll}
e_{3}: \quad y_{1} \frac{\partial F}{\partial y_{1}}+x_{2} \frac{\partial F}{\partial x_{2}}+y_{2} \frac{\partial F}{\partial y_{2}}=F, \\
e_{4}: \quad\left(-F+b_{4} x_{2}\right) \frac{\partial F}{\partial y_{1}}-\varepsilon y_{2} \frac{\partial F}{\partial x_{2}}+\varepsilon x_{2} \frac{\partial F}{\partial y_{2}}=y_{1}+d_{4} x_{2}+c_{4} y_{2}, \\
e_{5}: \quad y_{2} \frac{\partial F}{\partial y_{1}}=-\varepsilon x_{2} .
\end{array}
$$

Решение уравнения, отвечающего полю $e_{3}$, имеет вид

$$
F=y_{1} G\left(\frac{x_{2}}{y_{1}}, \frac{y_{2}}{y_{1}}\right) .
$$

Аналитическая функция $G\left(t_{1}, t_{2}\right)$ определяется далее из $e_{5}$-уравнения в виде

$$
G=-\frac{t_{1}}{t_{2}}+t_{1} \varphi\left(\frac{t_{1}}{t_{2}}\right)
$$

где $\varphi(\xi)$ - произвольная аналитическая функция. Ее мы находим из последнего уравнения системы (7.9), которое с учетом введения новых переменных принимает вид

$$
\left(1+\xi^{2}\right) \varphi^{\prime}(\xi)+\left(\frac{1}{\xi}-\xi\right) \varphi(\xi)=b_{4} \frac{1}{\xi}+\varepsilon d_{4}+\varepsilon c_{4} \xi
$$

Вводя вспомогательные параметры

$$
P=\frac{1}{2}\left(b_{4}+\varepsilon c_{4}\right), \quad R=\frac{1}{2}\left(b_{4}-\varepsilon c_{4}\right), \quad D=-\frac{\varepsilon d_{4}}{2},
$$


решение этого ОДУ (которое можно найти как «вручную», так и с помощью пакетов символьной математики) запишем в виде

$$
\varphi(\xi)=\left(P \operatorname{arctg} \xi+\frac{R \xi-D}{2\left(1+\xi^{2}\right)}+N\right) \frac{1+\xi^{2}}{\xi},
$$

где $N$ - произвольная константа интегрирования. Тогда уравнениями искомых однородных поверхностей являются

$$
v y_{2}+x_{2} y_{1}=N\left|z_{2}\right|^{2}+x_{2}\left(R y_{2}+D x_{2}\right)+P\left|z_{2}\right|^{2} \arg \left(z_{2}\right) .
$$

Заметим теперь, что это уравнение можно переписать в виде

$$
\left(v-R x_{2}-N y_{2}\right) y_{2}+x_{2}\left(y_{1}-D x_{2}-N x_{2}\right)=P\left|z_{2}\right|^{2} \arg \left(z_{2}\right) .
$$

Тогда замена переменных $z_{1}^{*}=z_{1}-i(N+D) z_{2}, w^{*}=w-(N+i R) z_{2}$ оставляет в правой части уравнения (7.10) лишь одно последнее слагаемое и приводит его к виду

$$
v y_{2}+x_{2} y_{1}=P\left|z_{2}\right|^{2} \arg \left(z_{2}\right) .
$$

При этом можно считать, что параметр $P$ принимает лишь значения 0 или 1 , так как умножение переменных $z_{1}, w$ на любой ненулевой вещественный множитель равносильно делению на этот множитель правой части уравнения (7.11) (при сохранении его вида).

Остается заметить, что это уравнение имеет индефинитную невырожденную форму Леви. Процедура его приведения к нормальной форме Мозера, относительно легко реализуемая, например, по схеме работы [9], дает тождественно нулевой многочлен $N_{220}$. Это означает омбиличность поверхности (7.11), то есть ее голоморфную сводимость к квадрике $v=\left|z_{1}\right|^{2}-\left|z_{2}\right|^{2}$. Предложение 7.2 доказано.

\section{СПИСОК ЛИТЕРАТУРЫ}

1. Акопян P. C., Лобода A. В. О голоморфных реализациях нильпотентных алгебр Ли// в кн.: Современные методы и проблемы математической гидродинамики-2018/ Междунар. науч. конф. (Воронежский государственный университет, 3-8 мая 2018 г.). - Воронеж: ВГПУ, 2018. - С. 200-204.

2. Атанов A. В., Лобода А. В. Голоморфные реализации разложимых пятимерных алгебр Ли// в кн.: Современные методы теории функций и смежные проблемы/ Воронежская зимняя математическая школа (28 января - 2 февраля 2019 г.). - Воронеж: ВГУ, 2019. - С. 24-26.

3. Атанов А. В., Лобода А. В., Суковых В. И. О голоморфной однородности вещественных гиперповерхностей общего положения в $\mathbb{C}^{3} / /$ Тр. Мат. ин-та им. В. А. Стеклова РАН. - 2017. - 298. C. $20-41$.

4. Бишоп P., Криттенден Р. Геометрия многообразий. - М.: Мир, 1967.

5. Ежов В. В., Лобода А. В., Шмальи Г. Каноническая форма многочлена четвертой степени в нормальном уравнении вещественной гиперповерхности в $\mathbb{C}^{3} / /$ Мат. заметки. -1999 . - 66, № 4. - С. 624-626.

6. Исаев А. В., Мищенко М. А. Классификация сферических трубчатых гиперповерхностей, имеющих в сигнатуре формы Леви один минус// Изв. АН СССР. Сер. мат. - 1988. - 52, № 6. - С. 1123-1153.

7. Лагно В. И., Спичак С. В., Стогний В. И. Симметрийный анализ уравнений эволюционного типа. М.-Ижевск: Ин-т комп. исслед., 2004.

8. Лобода $A$. B. О размерности группы, транзитивно действующей на гиперповерхности в $\mathbb{C}^{3} / /$ Функц. анал. прилож. - 1999. - 33, № 1. - С. 68-71.

9. Лобода А. В. Однородные вещественные гиперповерхности в $\mathbb{C}^{3}$ с двумерными группами изотропии// Тр. Мат. ин-та им. В. А. Стеклова РАН. - 2001. - 235. - С. 114-142.

10. Лобода $A$. В. Однородные строго псевдовыпуклые гиперповерхности в $\mathbb{C}^{3}$ с двумерными группами изотропии // Мат. сб. - 2001. - 192, № 12. - С. 3-24.

11. Лобода А. В. Всякая голоморфно-однородная трубка в $\mathbb{C}^{2}$ имеет аффинно-однородное основание// Сиб. мат. ж. - 2001. - 42, № 6. - С. 1335-1339.

12. Лобода $A$. В. Об определении однородной строго псевдовыпуклой гиперповерхности по коэффициентам ее нормального уравнения// Мат. заметки. - 2003. - 73, № 3. - С. 453-456.

13. Мубаракзянов Г. М. О разрешимых алгебрах Ли// Изв. вузов. Мат. - 1963. - № 1. - С. 114-123. 
14. Мубаракзянов Г. М. Классификация вещественных структур алгебр Ли пятого порядка// Изв. вузов. Мат. - 1963. - № 3. - С. 99-106.

15. Шабат Б. В. Введение в комплексный анализ. Функции нескольких переменных. - М.: Наука, 1985.

16. Beloshapka V. K., Kossovskiy I. G. Homogeneous hypersurfaces in $\mathbb{C}^{3}$, associated with a model CR-cubic// J. Geom. Anal. - 2010. - 20, № 3. - P. 538-564.

17. Cartan E. Sur la géométrie pseudoconforme des hypersurfaces de deux variables complexes: I// Ann. Math. Pura Appl. — 1932. — 11, № 4. - P. 17-90.

18. Chern S. S., Moser J. K. Real hypersurfaces in complex manifolds// Acta Math. — 1974. — 133. P. 219-271.

19. Dadok J., Yang P. Automorphisms of tube domains and spherical hypersurfaces// Am. J. Math. - 1985. - 107, № 4. - P. 999-1013.

20. Doubrov B., Komrakov B., Rabinovich M. Homogeneous surfaces in the three-dimensional affine geometry// in: Geometry and Topology of Submanifolds. — World Scientific, 1996. — 8. - P. 168-178.

21. Doubrov B., Medvedev A., The D. Homogeneous Levi nondegenerate hypersurfaces in $\mathbb{C}^{3} /$ arXiv: 1711.02389v1.

22. Fels G., Kaup W. Classification of Levi degenerate homogeneous CR-manifolds in dimension 5// Acta Math. - 2008. - 201. - P. 1-82.

Артем Викторович Атанов

Воронежский государственный университет

E-mail: atanov.cs@gmail.com

Александр Васильевич Лобода

Воронежский государственный технический университет

E-mail: lobvgasu@yandex.ru 